Series A

\author{
I. MATHEMATICA
}

415

\title{
EIN NEUER ZUGANG ZUR THEORIE DER BESSELFUNKTIONEN IN MEHREREN DIMENSIONEN
}

VON

CLAUS MÜLLER

H E L S I K I 1968

S U O M A L A I N E N T I E D A K A T E M A 
KESKUSKIRJAPAINO

HELSINKI 1968 


\section{Ein neuer Zugang zur Theorie der Besselfunktionen in mehreren Dimensionen}

Im klassischen Aufbau der Theorie der Besselfunktionen wird davon ausgegangen, die Schwingungsgleichung

$$
\Delta U+U=0
$$

durch Einführung von Polarkoordinaten zu separieren und dann die entstehenden gewöhnlichen Differentialgleichungen zu integrieren [7].

Ein anderer Weg, der zunächst von Poisson und später von Sommerfeld beschritten wurde, geht von der Bemerkung aus, dass für jeden reellen oder komplexen Wert $\alpha$

$$
e^{i\left(x_{1} \cos \alpha+x_{2} \sin \alpha\right)}
$$

eine Lösung der Helmholtzschen Gleichung in zwei Dimensionen ist. Durch geeignete Superposition dieser Funktionen lassen sich dann weitere partikuläre Lösungen der Schwingungsgleichung finden. So erhält man durch

$$
V_{n}\left(x_{1}, x_{2}\right)=\frac{i^{-n}}{2 \pi} \int_{-\pi}^{\pi} e^{i\left(x_{1} \cos \alpha+x_{2} \sin \alpha\right)} e^{i n \alpha} d x
$$

eine Funktion, die sich nach Einführung der Polarkoordinaten

$$
x_{1}=r \cos \varphi ; \quad x_{2}=r \sin \varphi
$$

in der Form

$$
V_{n}\left(x_{1}, x_{2}\right)=J_{n}(r) e^{i n \varphi}
$$

mit

$$
J_{n}(r)=\frac{i^{-n}}{2 \pi} \int_{-\pi}^{+\pi} e^{i r \cos \alpha} e^{i n \alpha} d \alpha
$$

schreiben lässt. 
A. Sommerfeld führte 1896 [5] die Funktionen

$$
U_{n}^{(1)}\left(x_{1}, x_{2}\right)=\frac{i^{-n}}{\pi} \int_{-\frac{\pi}{2}+\psi+i \infty}^{\frac{\pi}{2}+\psi^{\prime-i \infty}} e^{i\left(x_{1} \cos \alpha+x_{2} \sin \alpha\right)} e^{i n \alpha} d \alpha
$$

und

$$
U_{n}^{(2)}\left(x_{1}, x_{2}\right)=\frac{i^{-n}}{\pi} \int_{\frac{\pi}{2}+\psi^{-i \infty}}^{\frac{3 \pi}{2}+\psi+i \infty} e^{i\left(x_{1} \cos \alpha+x_{2} \sin \alpha\right)} e^{i n \alpha} d \alpha
$$

ein, wobei diese Darstellungen für $|\varphi-\psi|<\pi / 2$ gelten. Es gilt dann weiterhin

$$
\begin{aligned}
& U_{n}^{(1)}\left(x_{1}, x_{2}\right)=H_{n}^{(1)}(r) e^{i n_{\varphi}} \\
& U_{n}^{(2)}\left(x_{1}, x_{2}\right)=H_{n}^{(2)}(r) e^{i n_{\varphi}}
\end{aligned}
$$

mit

$$
\begin{aligned}
H_{n}^{(1)}(r)= & \frac{i^{-n}}{\pi} \int_{-\frac{\pi}{2}+i \infty}^{\frac{\pi}{2}-i \infty} e^{i r \cos \alpha} e^{i n \alpha} d x, \\
H_{n}^{(2)}(r)= & \frac{i^{-n}}{\pi} \int_{\frac{\pi}{2}-i \infty}^{\frac{3 \pi}{2}+i \infty} e^{i r \cos \alpha} e^{i n \alpha} d x .
\end{aligned}
$$

Bei dieser Einführung entstehen die Besselfunktionen $J_{n}(r)$ und die Hankelfunktionen $H_{n}^{(1)}(r), H_{n}^{(2)}(r)$ in physikalischer Deutung als Superpositionen von ebenen Wellen der Form

$$
e^{i\left(x_{1} \cos \alpha+x_{2} \sin \alpha\right)},
$$

mit reellen Wellenrichtungen im Falle der Besselfunktionen, und mit komplexen Wellenrichtungen im Falle der Hankelfunktionen.

Während die Darstellungen der Besselfunktionen für alle Punkte $x=\left(x_{1}, x_{2}\right)$ gelten, sind die Darstellungen der Hankelfunktionen jeweils nur in Halbräumen gültig.

Aus physikalischer Intuition sind analoge Darstellungen auch in Räumen höherer Dimension zu erwarten. Die mathematische Theorie dieser Zusammenhänge wird hier erstmalig geliefert. 
Dabei wird einerseits die Theorie der Kugelfunktionen in einer kürzlich entwickelten Form benutzt [1], und es wird andererseits eine Technik der Deformation der Integrationsmannigfaltigkeiten in mehreren komplexen Dimensionen verwandt, die in dieser Form neu sein dürfte.

Neben einem neuen Zugang zur Theorie der Bessel- und Hankelfunktionen wird dabei eine Zusammenfassung vieler Beziehungen erreicht, die bisher als Einzelergebnisse erschienen.

Zur Vereinheitlichung der Darstellung wurde auch die reelle Theorie der Besselfunktionen aufgenommen, wie sie wohl zuerst von G. Herglotz konzipiert wurde [2]. Durch die Gegenüberstellung der reellen Theorie der Besselfunktionen und der komplexen Theorie der Hankelfunktionen dürften - ähnlich wie in der Sommerfeldschen Theorie - die Merkmale der komplex-analytischen Methode besonders hervortreten.

Herrn C. Engeln möchte ich für seine Hilfe bei der Anfertigung des Manuskriptes und seine Anregungen zur Verbesserung der Darstellung recht herzlich danken.

Über die speziellen Ergebnisse dieser Arbeit hinaus erweist sich vermutlich die Untersuchung spezieller Funktionen mit Hilfe der mehrdimensionalen komplexen Analysis als geeignetes Mittel, Kalkül und Technik dieser Disziplin weiterzuentwickeln. Die vorliegende Arbeit ist auch als Beitrag zu diesem Fragenkreis gedacht.

\section{§ 1. Bezeichnungen}

Mit $E_{q}$ bezeichnen wir den euklidischen Raum von $q$ reellen Dimensionen, seine Elemente mit $x, y, z$ oder $x_{(q)}, y_{(q)}, z_{(q)}$, wenn die Angabe der Dimension wichtig ist. In der Komponentenzerlegung ist dann

$$
x_{(q)}=\left(x_{1}, x_{2}, \ldots, x_{q}\right) .
$$

Skalarprodukt und absoluter Betrag werden, wie üblich, durch

$$
x \cdot y=x_{1} y_{1}+\cdots+x_{q} y_{q} ; \quad x^{2}=x \cdot x ; \quad|x|=\sqrt{x^{2}}
$$

eingeführt. Mit $\Omega_{q}$ werde die Einheitssphäre $x^{2}=1$ in $E_{q}$ bezeichnet. Ihre Elemente nennen wir $\xi, \eta, \zeta$.

Dann gilt für $x \in E_{q}, \quad x \neq 0$

$$
x=r \xi ; \quad r=|x| ; \quad \xi \in \Omega_{q},
$$

wobei $\xi$ der eindeutig bestimmte Richtungsvektor $\mathrm{zu} \quad x$ ist.

Es sei $\varepsilon_{1}, \varepsilon_{2}, \ldots, \varepsilon_{q}$ die übliche orthonormierte Basis von $E_{q}$. Die Gesamtheit der Vektoren 


$$
x=x_{1} \varepsilon_{1}+x_{2} \varepsilon_{2}+\cdots+x_{q} \varepsilon_{q}
$$

mit reellen oder komplexen Komponenten $x_{1}, x_{2}, \ldots, x_{q}$ bezeichnen wir als $C_{q}$. Die Menge aller $x \in C_{q}$ mit $x^{2}=1$ nennen wir $\Omega_{q}^{*}$.

Dem reellen Raum $E_{q}$ legen wir die kanonische Orientierung zugrunde und bezeichnen mit

$$
d \pi_{q}(x)=d x_{1} \wedge d x_{2} \wedge \ldots \wedge d x_{q}
$$

das Volumenelement. Es sei

$$
\begin{aligned}
& d \omega_{q}(x)= \\
& \quad \begin{aligned}
x_{1} d x_{2} \wedge \ldots \wedge & d x_{q}-x_{2} d x_{1} \wedge d x_{3} \wedge \ldots \wedge d x_{q}+\ldots \\
& +(-1)^{q-1} x_{q} d x_{1} \wedge d x_{2} \wedge \ldots \wedge d x^{q-1} .
\end{aligned}
\end{aligned}
$$

Dann ist diese Differentialform vom Grade $q-1$ auf $\Omega_{q}$ gleich dem orientierten Flächenelement. Beide Differentialformen lassen sich ohne weiteres ins Komplexe übertragen.

Bezeichnen wir mit

$$
|a, b, \ldots|_{(q)}
$$

eine $q$-dimensionale Determinante mit den Spalten $a, b, \ldots$, und setzen wir bei Vorliegen einer Parameterdarstellung

$$
x=x\left(u_{1}, u_{2}, \ldots, u_{r}\right) \quad \text { mit } r=q \text { oder } r=q-1
$$

für die Ableitungen

$$
\frac{\partial}{\partial u^{j}} x=x_{\mid j}
$$

so wird

$$
d \pi_{q}(x)=\left|x_{\mid 1}, x_{\mid 2}, \ldots, x_{\mid q}\right|_{(q)} d u_{1} \wedge d u_{2} \wedge \ldots \wedge d u_{q}
$$

und im Falle einer $(q-1)$-dimensionalen Parameterdarstellung

$$
d \omega_{q}(x)=\left|x, x_{\mid 1}, x_{\mid 2}, \ldots, x_{q-1}\right|_{(q)} d u_{1} \wedge d u_{2} \wedge \ldots \wedge d u_{q-1} .
$$

Die Differentialform $d \pi_{q}(x)$ wird immer durch eine $q$-dimensionale reelle Parameterdarstellung und die Differentialform $d \omega_{q}(x)$ durch eine $(q-1)$-dimensionale reelle Parameterdarstellung ausgedrückt werden.

Wir wollen nun einige einfache Beziehungen dieser Differentialformen ableiten. Zunächst entnimmt man aus (5) und (6)

$$
d \pi_{q}=d \pi_{q-1} \wedge d x_{q}
$$

und

$$
d \omega_{g}=d \omega_{q-1} \wedge d x_{q}+(-1)^{q-1} x_{q} d \pi_{q-1} .
$$


Es sei nun $\Lambda(x)$ eine differenzierbare Funktion von $x_{1}, x_{2}, \ldots, x_{q}$. Dann ist

$$
d \pi_{q}(\Lambda x)=\prod_{i=1}^{q}\left(\Lambda d x_{i}+x_{i} d \Lambda\right)
$$

wobei das Produkt im Sinne der alternierenden Multiplikation unter Beachtung der natürlichen Reihenfolge $1, \ldots, q$ zu verstehen ist. Es wird damit

$$
d \pi_{q}(x)=\Lambda^{q} d \pi_{q}(x)+\Lambda^{q-1} d \Lambda \wedge d \omega_{q}(x),
$$

wie sich durch Ausrechnen leicht bestätigt.

Aus (11) entnehmen wir weiterhin

$$
d \omega_{q}(\Lambda x)=\Lambda^{q} d \omega_{q}(x) .
$$

Wir wollen nun Polarkoordinaten einführen und setzen $x=r \xi$ mit $\xi^{2}=1$. Dann ist

$$
\xi_{1} d \xi_{1}+\xi_{2} d \xi_{2}+\cdots+\xi_{q} d \xi_{q}=0
$$

und folglich

$$
d \pi_{q}(\xi)=0
$$

Aus (15) folgt daher

$$
d \pi_{q}(r \xi)=r^{q-1} d r \wedge d \omega_{q}(\xi) .
$$

Bezeichnen wir mit $d \lambda(x)$ eine der beiden Differentialformen (10) oder (11), so ist offenbar für jede konstante Matrix $A$

$$
d \lambda(A x)=\operatorname{det} A d \lambda(x) .
$$

Es gilt somit insbesondere

Lemma 1. Es sei A eine eigentlich orthogonale Matrix. Dann ist

$$
d \pi_{q}(A x)=d \pi_{q}(x)
$$

und

$$
d \omega_{q}(A x)=d \omega_{q}(x)
$$

Wir wollen nun zu Parameterdarstellungen von $\Omega_{q}$ und Berechnungen der $d \omega_{q}$ auf $\Omega_{q}$ übergehen.

Jeden Einheitsvektor $\xi_{(q)}$ können wir in der Form

$$
\xi_{(q)}=t \varepsilon_{q}+\sqrt{1-t^{2}} \xi_{(q-1)} ; \quad-1 \leqq t \leqq 1 ; \quad \xi_{(q-1)} \in \Omega_{q-1}
$$

darstellen. Aus (13) folgt nun 
(22) $d \omega_{q}\left(t \varepsilon_{q}+\sqrt{1-t^{2}} \xi_{(q-1)}\right)$

$$
=d \omega_{q-1}\left(\sqrt{1-t^{2}} \xi_{(q-1)}\right) \wedge d t+(-1)^{q-1} t d \pi_{q-1}\left(\sqrt{1-t^{2}} \xi_{(q-1)}\right) .
$$

Nach (15) und (18) ist

$$
\begin{gathered}
d \pi_{q-1}\left(\sqrt{1-t^{2}} \xi_{(q-1)}\right)=-t\left(1-t^{2}\right)^{\frac{q-3}{2}} d t \wedge d \omega_{q-1}\left(\xi_{(q-1)}\right) \\
=(-1)^{q-1} t\left(1-t^{2}\right)^{\frac{q-3}{2}} d \omega_{q-1}\left(\xi_{(q-1)}\right) \wedge d t
\end{gathered}
$$

und nach (16)

$$
d \omega_{q-1}\left(\sqrt{1-t^{2}} \xi_{(q-1)}\right)=\left(1-t^{2}\right)^{\frac{q-1}{2}} d \omega_{q-1}\left(\xi_{(q-1)}\right) .
$$

Damit folgt aus (22)

$$
\begin{aligned}
& d \omega_{q}\left(t \varepsilon_{q}+\sqrt{1-t^{2}} \xi_{(q-1)}\right) \\
& =\left(1-t^{2}\right)^{\frac{q-3}{2}}\left[\left(1-t^{2}\right)+t^{2}\right] d \omega_{q-1}\left(\xi_{(q-1)}\right) \wedge d t
\end{aligned}
$$

und wir finden

Lemma 2. Es sei

$$
\xi_{(q)}=t \varepsilon_{q}+\sqrt{1-t^{2}} \xi_{(q-1)} ; \quad-1 \leqq t \leqq 1 ; \quad \xi_{(q-1)} \in \Omega_{q-1},
$$

eine Parameterdarstellung von $\Omega_{q}$. Dann ist

$$
d \omega_{q}\left(\xi_{(q)}\right)=\left(1-t^{2}\right)^{\frac{q-3}{2}} d \omega_{q-1}\left(\xi_{(q-1)}\right) \wedge d t .
$$

Bezeichnen wir mit

$$
\omega_{q}=\int_{\Omega_{q}} d \omega_{q}(\xi)
$$

das Mass der Einheitssphäre $\Omega_{q}$ in $E_{q}$, so wird

$$
\omega_{q}=\int_{-1}^{+1} \int_{\Omega_{q-1}}\left(1-t^{2}\right)^{\frac{q-3}{2}} d \omega_{q-1} \wedge d t=\omega_{q-1} \int_{-1}^{+1}\left(1-t^{2}\right)^{\frac{q-3}{2}} d t .
$$

Setzen wir $\omega_{1}=2$, so wird für $q=2,3, \ldots$

$$
\omega_{q}=\frac{\sqrt{\pi} \Gamma\left(\frac{q-1}{2}\right)}{\Gamma\left(\frac{q}{2}\right)} \omega_{q-1}=2 \frac{\pi^{\frac{q}{2}}}{\Gamma\left(\frac{q}{2}\right)} .
$$




\section{§ 2. Theorie der Kugelfunktionen}

In diesem Abschnitt werden wir eine kurze Darstellung der für den hier vorliegenden Fragenkreis wichtigsten Ergebnisse aus der Theorie der Kugelfunktionen geben. Ausführlichere Darstellungen liegen an anderer Stelle vor [1].

Es sei

$$
\Delta_{(q)}=\left(\frac{\partial}{\partial x_{1}}\right)^{2}+\cdots+\left(\frac{\partial}{\partial x_{q}}\right)^{2}
$$

der Laplace-Operator in $q$ Dimensionen.

Es sei weiterhin $H_{n}(x)$ ein homogenes Polynom der Veränderlichen $\left(x_{1}, \ldots, x_{q}\right)$ vom Grade $n$, das

$$
\Delta_{(g)} H_{n}(x)=0
$$

erfüllt. Dann ist mit der Darstellung $x=r \xi$

$$
H_{n}(x)=H_{n}(r \xi)=r^{n} H_{n}(\xi) \text {. }
$$

Wir nennen nun

$$
S_{n}(q ; \xi)=S_{n}(\xi)=H_{n}(\xi)
$$

eine Kugelfunktion der Ordnung $n$ in $q$ Dimensionen.

Jedes Polynom $H_{n}(x)$ kann in der Form

$$
H_{n}\left(x_{1}, \ldots, x_{q}\right)=\sum_{j=0}^{n}\left(x_{q}\right)^{j} A_{n-j}\left(x_{1}, \ldots, x_{q-1}\right)
$$

dargestellt werden, wobei die Koeffizienten $A_{n-j}$ Polynome vom Grade $n-j$ in $x_{1}, \ldots, x_{q-1}$ sind.

Mit

$$
\Delta_{(q)}=\left(\frac{\partial}{\partial x_{q}}\right)^{2}+\Delta_{(q-1)}
$$

wird

$$
\left.=\sum_{j=0}^{n-2}\left[(j+2)(j+1) A_{n-j-2}+\Delta_{(g-1)} A_{n-j}\right]\left(x_{q}\right)^{j}+x_{q} \Delta_{(q-1)} A_{1}+\right\lrcorner_{(q-1)} A_{0}=0,
$$

wobei die letzten beiden Glieder gleich Null sind. Für die Koeffizientenfunktionen der homogenen harmonischen Polynome gilt daher

$$
\Delta_{(q-1)} A_{n-j}=-(j+2)(j+1) A_{n-j-2},
$$


so dass nur die Polynome $A_{n}$ und $A_{n-1}$ frei wählbar sind. Die Zahl der in diesen beiden Polynomen verfügbaren Koeffizienten gibt folglich auch die Anzahl der linear unabhängigen Kugelfunktionen an. Wir bezeichnen sie mit $N(q, n)$ und verzichten hier auf ihre explizite Berechnung. Aus einer einfachen Abzählung ergibt sich $N(q, n)=O\left(n^{q-2}\right)$.

Wir wollen nun eine Kugelfunktion mit Rotationssymmetrie bestimmen und suchen dazu eine Funktion $L_{n}(x)$ mit den folgenden Eigenschaften:

1. $L_{n}(x)$ ist ein homogenes, harmonisches Polynom vom Grade $n$ in $q$ Dimensionen.

2. $L_{n}(D x)=L_{n}(x)$ für alle orthogonalen Transformationen $D$ mit $D \varepsilon_{q}=\varepsilon_{q}$.

3. $L_{n}\left(\varepsilon_{q}\right)=1$.

Aus der zweiten Bedingung folgt, dass für das Polynom $L_{n}(x)$ die Koeffizientenfunktionen $A_{n-j}$ nur von $x_{1}^{2}+\cdots+x_{q-1}^{2}$ abhängen. Da sie gleichzeitig homogene Polynome sein müssen, ergibt sich

$$
A_{n-j}=\left\{\begin{array}{ll}
0 & \text { für } n-j \text { ungerade } \\
c\left(x_{1}^{2}+\cdots+x_{q-1}^{2}\right)^{k} & \text { für } n-j=2 k
\end{array} .\right.
$$

Von den beiden Zahlen $n$ und $n-1$ ist stets eine gerade, so dass $L_{n}(x)$ durch die erste und die zweite Bedingung bis auf einen Faktor eindeutig festgelegt ist. Nach (5) ist

$$
L_{n}\left(\varepsilon_{q}\right)=A_{0},
$$

wobei dieser Koeffizient proportional zu dem noch frei verfügbaren Faktor ist. Durch die dritte Bedingung wird die Funktion $L_{n}(x)$ damit dann eindeutig bestimmt.

Setzen wir

$$
x_{(q)}=r\left(t \varepsilon_{q}+\sqrt{1-t^{2}} \xi_{(q-1)}\right),
$$

so wird

$$
L_{n}(x)=r^{n} L_{n}\left(t \varepsilon_{q}+\sqrt{1-t^{2}} \xi_{(q-1)}\right)=r^{n} P_{n}(q ; t),
$$

wobei $P_{n}(q ; t)$ ein Polynom vom Grade $n$ in $t$ ist, das den Bedingungen

$$
P_{n}(q ; 1)=1 ; \quad P_{n}(q ;-1)=(-1)^{n}
$$

genügt.

Mit Hilfe dieser Funktionen können wir nun das für unsere weiteren Überlegungen wichtige Additionstheorem beweisen. Wir nehmen dazu an, dass ein orthonormiertes System von Kugelfunktionen $S_{n, j}(\xi)$ mit $j=1,2, \ldots, N$ ausgewählt wurde. 
Es sei nunmehr $A$ eine orthogonale Transformationsmatrix. Dann ist mit $S_{n}(\xi)$ auch $S_{n}(A \xi)$ eine Kugelfunktion der Ordnung $n$, da mit $H_{n}(x)$ auch $H_{n}(A x)$ ein homogenes, harmonisches Polynom vom Grade $n$ ist. Zu jedem orthogonalen $A$ gibt es daher Koeffizienten $C_{j k}$ so, dass

$$
S_{n, j}(A \xi)=\sum_{k=1}^{N} C_{j k} S_{n, k}(\xi)
$$

ist. Wir betrachten nun

$$
\delta_{j l}=\int_{\Omega_{q}} S_{n, j}(\xi) S_{n, l}(\xi) d \omega_{q}(\xi)=\int_{A \Omega_{q}} S_{n, j}(\xi) S_{n, l}(\xi) d \omega_{q}(\xi)(\operatorname{det} A) .
$$

Diese Beziehung bringt zum Ausdruck, dass $\Omega_{q}$ bei einer orthogonalen Transformation $A$ in sich übergeht, wobei die Orientierung erhalten bleibt, wenn $\operatorname{det} A=1$ ist, für $\operatorname{det} A=-1$ jedoch geändert wird. Das letzte Integral können wir auch in der Form

$$
\int_{\Omega_{q}} S_{n, j}(A \xi) S_{n, l}(A \xi) d \omega_{q}(A \xi)
$$

schreiben. Es ist aber nach Lemma 1:

$$
d \omega_{q}(A \xi)=d \omega_{q}(\xi)(\operatorname{det} A),
$$

so dass sich aus (14) und der Orthogonalität der $S_{n, j}$ schliesslich

$$
\delta_{j l}=\sum_{k=1}^{N} C_{j k} C_{l k}
$$

ergibt. Die Koeffizienten $C_{j k}$ bilden folglich eine orthogonale Matrix, und es gilt daher auch

$$
\delta_{j l}=\sum_{k=1}^{N} C_{k j} C_{k l} .
$$

Bilden wir nun zu zwei Einheitsvektoren $\xi$ und $\eta$ die Summe

$$
\sum_{j=1}^{N} S_{n, j}(\xi) S_{n, j}(\eta)=F(\xi, \eta)
$$

so genügt diese Funktion für alle orthogonalen $A$ der Bedingung

$$
F(A \xi, A \eta)=F(\xi, \eta) .
$$

Damit muss $F(\xi, \eta)$ eine Funktion sein, die nur vom Skalarprodukt abhängt. Es gilt also

$$
F(\xi, \eta)=G(\xi \cdot \eta) .
$$


Bei festem $\eta$ ist $G(\xi \cdot \eta)$ eine Kugelfunktion der Ordnung $n$ in $\xi$. Sie ist symmetrisch bezüglich aller Drehungen oder Spiegelungen, die $\eta$ festlassen.

Es gilt also

$$
G(\xi \cdot \eta)=c_{n} P_{n}(q ; \xi \cdot \eta)
$$

Zur Bestimmung von $c_{n}$ setzen wir $\xi=\eta$ und integrieren über $\Omega_{q}$. Dann folgt aus

$$
\int_{\Omega_{q}} \sum_{j=1}^{N}\left(S_{n, j}(\xi)\right)^{2} d \omega_{q}(\xi)=c_{n} \int_{\Omega_{q}} d \omega_{q}
$$

schliesslich

$$
N(q ; n)=c_{n} \omega_{q}
$$

Wir erhalten daher

Satz 1. Es sei $\left\{S_{n, j}(\xi)\right\}$ ein orthonormiertes System von Kugelfunktionen der Ordnung $n$ und Dimension $q$. Dann ist für beliebige $\xi$ und $\eta$ aus $\Omega_{q}$

$$
\sum_{j=1}^{N} S_{n, j}(\xi) S_{n, j}(\eta)=\frac{N(q ; n)}{\omega_{q}} P_{n}(q ; \xi \cdot \eta)
$$

Aus dieser Beziehung folgt unmittelbar

Satz 2. Es sei $S_{n}(\xi)$ eine Kugelfunktion der Ordnung n. Dann ist

$$
\frac{N}{\omega_{q}} \int_{\Omega_{q}} P_{n}(q ; \xi \cdot \eta) S_{n}(\eta) d \omega_{q}(\eta)=S_{n}(\xi) .
$$

Wir wollen nun eine weitere wichtige Beziehung herleiten, die den Schlüssel zur Übertragung der Poissonschen Definition der Besselfunktionen liefert.

Es sei $f(t)$ eine für $-1 \leqq t \leqq 1$ stetige Funktion. Mit zwei Einheitsvektoren $\xi$ und $\zeta$ bilden wir

$$
\int_{\Omega_{q}} f(\xi \cdot \eta) P_{n}(q ; \zeta \cdot \eta) d \omega_{q}(\eta)=F(\xi, \zeta) \text {. }
$$

Dann ist für jede orthogonale Matrix A

$$
\begin{gathered}
F(A \xi, A \zeta)=\int_{\Omega_{q}} f(A \xi \cdot \eta) P_{n}(q ; A \zeta \cdot \eta) d \omega_{q}(\eta) \\
\quad=\int_{A} f(A \xi \cdot \eta) P_{n}(q ; A \zeta \cdot \eta) d \omega_{q}(\eta)(\operatorname{det} A) .
\end{gathered}
$$


Dies entspricht der schon einmal in (15) durchgeführten Umformung. Wir können nun weiter schliessen

$$
F(A \xi, A \eta)=\int_{\Omega_{q}} f(A \xi \cdot A \eta) P_{n}(q ; A \zeta \cdot A \eta) d \omega_{q}(A \eta)(\operatorname{det} A)
$$

da wir eine Transformation der Integrationsmannigfaltigkeit durch die gleiche Transformation der Integrationsvariablen ausdrücken können. Auf Grund der Invarianz des Skalarproduktes gegenüber Orthogonaltransformationen und der Transformationseigenschaft (17) folgt

$$
F(A \xi, A \zeta)=F(\xi, \zeta) \text {. }
$$

Damit kann auch diese Funktion nur vom Skalarprodukt $\xi \cdot \zeta$ abhängen. Da sie bezüglich $\zeta$ eine Kugelfunktion der Ordnung $n$ ist, folgt

$$
\int_{\Omega_{q}} f(\xi \cdot \eta) P_{n}(q ; \zeta \cdot \eta) d \omega_{q}(\eta)=\lambda_{n} P_{n}(q ; \xi \cdot \zeta)
$$

Zur Bestimmung von $\lambda_{n}$ setzen wir $\xi=\zeta=\varepsilon_{q}$ und

$$
\eta=\eta_{(q)}=t \varepsilon_{q}+\sqrt{1-t^{2}} \eta_{(q-1)} .
$$

Dann wird aus (30)

$$
\lambda_{n} P_{n}(q ; 1)=\hat{\lambda}_{n}=\int_{\Omega_{q}} f\left(\varepsilon_{q} \cdot \eta\right) P_{n}\left(q ; \varepsilon_{q} \cdot \eta\right) d \omega_{q}(\eta)
$$

und wir erhalten nach Lemma 2

$$
\lambda_{n}=\omega_{q-1} \int_{-1}^{+1} f(t) P_{n}(q ; t)\left(1-t^{2}\right)^{\frac{q-3}{2}} d t
$$

Multiplizieren wir nun noch beide Seiten von (30) mit einer Kugelfunktion $\left(N / \omega_{q}\right) S_{n}(\zeta)$ und integrieren bezüglich $\zeta$ über $\Omega_{q}$, so folgt nach Satz 2

Satz 3. Es sei $f(t)$ eine für $-1 \leqq t \leqq 1$ stetige Funktion und $S_{n}(\eta)$ eine Kugelfunktion der Ordnung $n$. Dann ist

$$
\int_{\Omega_{q}} f(\xi \cdot \eta) S_{n}(\eta) d \omega_{q}(\eta)=\lambda_{n} S_{n}(\xi)
$$

mit 


$$
\lambda_{n}=\omega_{q-1} \int_{-1}^{+1} f(t) P_{n}(q ; t)\left(1-t^{2}\right)^{\frac{q-3}{2}} d t .
$$

Diese auf Funk und Hecke zurückgehende Formel kombiniert die Orthogonalinvarianz der Kugel mit dem Additionstheorem der Kugelfunktionen.

Betrachten wie zwei Kugelfunktionen $S_{n}(\xi)$ und $S_{m}(\xi)$, so gilt nach der Greenschen Formel für die zugehörigen harmonischen Polynome $H_{n}(x)$ und $H_{m}(x)$

$$
\begin{aligned}
0 & =\int_{|x| \leqq 1}\left(H_{n} \Delta H_{m}-H_{m} \Delta H_{n}\right) d \pi_{q}(x) \\
& =\int_{|x|=1}\left(H_{n} \frac{\partial}{\partial r} H_{m}-H_{m} \frac{\partial}{\partial r} H_{n}\right) d \omega_{q}(x) .
\end{aligned}
$$

Für $r=1$ ist aber $(l=0,1, \ldots)$

$$
\frac{\partial}{\partial r} H_{l}(x)=l H_{l}(x)=l S_{l}(\xi) \text {. }
$$

Aus (34) folgt daher

$$
(m-n) \int_{\Omega_{q}} S_{n}(\xi) S_{m}(\xi) d \omega_{q}(\xi)=0 .
$$

Damit sind Kugelfunktionen verschiedener Ordnung stets orthogonal zueinander. Wenden wir dieses Ergebnis auf die verallgemeinerten Legendre-Funktionen $L_{n}(x)$ an, so ergibt sich für $n \neq m$

$$
0=\int_{\Omega_{q}} L_{n}(\xi) L_{m}(\xi) d \omega_{q}(\xi)=\omega_{q-1} \int_{-1}^{+1} P_{n}(q, t) P_{m}(q, t)\left(1-t^{2}\right)^{\frac{q-3}{2}} d t .
$$

Die Funktionen $P_{n}(q, t)$ sind Polynome vom Grade $n$, die die Normierungsbedingung $P_{n}(q, 1)=1$ erfüllen. Sie sind daher durch die Eigenschaft (37) und die letzten beiden Eigenschaften eindeutig bestimmt.

Unter Benutzung dieser Tatsache ergibt sich die verallgemeinerte Formel von Rodrigues

$$
P_{n}(q, t)=\left(-\frac{1}{2}\right)^{n} \frac{\Gamma\left(\frac{q-1}{2}\right)}{\Gamma\left(n+\frac{q-1}{2}\right)}\left(1-t^{2}\right)^{\frac{3-q}{2}}\left(\frac{d}{d t}\right)^{n}\left(1-t^{2}\right)^{n+\frac{q-3}{2}} .
$$


Damit folgt für jede $n$-mal stetig differenzierbare Funktion $f(t)$

$$
\int_{-1}^{+1} f(t) P_{n}(q, t)\left(1-t^{2}\right)^{\frac{q-3}{2}} d t=\left(\frac{1}{2}\right)^{n} \frac{\Gamma\left(\frac{q-1}{2}\right)}{\Gamma\left(n+\frac{q-1}{2}\right)} \int_{-1}^{+1} f^{(n)}(t)\left(1-t^{2}\right)^{n+\frac{q-3}{2}} d t
$$

Im Hinblick auf die nun folgende Theorie der verallgemeinerten Besselfunktionen bilden wir speziell

$$
\begin{aligned}
& \int_{-1}^{+1} e^{i r t} P_{n}(q, t)\left(1-t^{2}\right)^{\frac{q-3}{2}} d t \\
& =\left(\frac{i r}{2}\right)^{n} \frac{\Gamma\left(\frac{q-1}{2}\right)}{\Gamma\left(n+\frac{q-1}{2}\right)} \int_{-1}^{+1} e^{i r t}\left(1-t^{2}\right)^{n+\frac{q-3}{2}} d t
\end{aligned}
$$

und erhalten daraus die Abschätzung

$$
\int_{-1}^{+1} e^{i r t} P_{n}(q, t)\left(1-t^{2}\right)^{\frac{q-3}{2}} d t \leqq\left(\frac{r}{2}\right)^{n} \frac{\Gamma\left(\frac{q-1}{2}\right) \Gamma\left(\frac{1}{2}\right)}{\Gamma\left(n+\frac{q}{2}\right)}
$$

Der klassische Aufbau der Theorie der Besselfunktionen stellt die Integrale (40) in den Mittelpunkt. Wir wollen hier, einem Gedanken von G. Herglotz folgend, den Zugang von Poisson und Sommerfeld verallgemeinern.

\section{§3. Die regulären Besselfunktionen}

Wir werden nun die Besselfunktionen und ihre Integraldarstellungen für ganzzahlige Ordnungen auf beliebig viele Dimensionen übertragen. Wir setzen dazu

$$
V_{n}(x)=\frac{i^{-n}}{\omega_{q}} \int_{\Omega_{q}} e^{i x \cdot \eta} S_{n}(\eta) d \omega_{q}(\eta)
$$

Mit Polarkoordinaten $x=r \xi$ wird daraus 


$$
V_{n}(r \xi)=\frac{i^{-n}}{\omega_{q}} \int_{\Omega_{q}} e^{i r \xi \bullet \eta} S_{n}(\eta) d \omega_{q}(\eta) .
$$

Nach Satz 3 ist folglich

$$
V_{n}(r \xi)=J_{n}(q ; r) S_{n}(\xi)
$$

wenn wir

$$
J_{n}(q ; r)=\frac{i^{-n} \omega_{q-1}}{\omega_{q}} \int_{-1}^{+1} e^{i r t} P_{n}(q ; t)\left(1-t^{2}\right)^{\frac{q-3}{2}} d t
$$

setzen.

Dies führt uns zu

Definition 1. Die Funktion

$$
J_{n}(q ; r)=\frac{i^{-n} \omega_{q-1}}{\omega_{q}} \int_{-1}^{+1} e^{i r t} P_{n}(q ; t)\left(1-t^{2}\right)^{\frac{q-3}{2}} d t
$$

heisst (reguläre) Besselfunktion der Ordnung $n$ und Dimension $q$.

Nach (2.41) erhalten wir bei festem $r$ für $n \rightarrow \infty$

$$
J_{n}(q ; r)=O\left(\frac{\left(\frac{r}{2}\right)^{n}}{\Gamma\left(n+\frac{q}{2}\right)}\right) .
$$

Die Definition 1 liefert mit Hilfe der Theorie der Kugelfunktionen eine grosse Zahl von speziellen Beziehungen, von denen hier nur einige als Beispiele angeführt seien.

Das System der orthonormierten Kugelfunktionen ist vollständig [1]. Konvergiert daher die verallgemeinerte Fourier-Reihe einer auf $\Omega_{q}$ stetigen Funktion, so stellt sie diese Funktion auch dar.

Wählen wir $e^{i r \xi \eta}$ mit festem Einheitsvektor $\eta$ als Beispiel, so sind die Entwicklungskoeffizienten bezüglich $S_{n, j}(\xi)$ nach (3) und (4) gleich

$$
\omega_{q} i^{n} J_{n}(q ; r) S_{n, j}(\eta) \text {. }
$$

Unter Beachtung des Additionstheorems (Satz 1) wird

$$
\begin{aligned}
& \omega_{q} \sum_{n=0}^{\infty} i^{n} J_{n}(q ; r) \sum_{j=1}^{N} S_{n, j}(\eta) S_{n, j}(\xi) \\
& =\sum_{n=0}^{\infty} i^{n} J_{n}(q ; r) N(q ; n) P_{n}(q ; \xi \cdot \eta) .
\end{aligned}
$$


Es ist aber bei festem $r$ für $n \rightarrow \infty$

$$
J_{n}(q, r) P_{n}(q ; \xi \cdot \eta) N(q ; n)=O\left(\left(\frac{r}{2}\right)^{n} \frac{n^{q-2}}{\Gamma\left(n+\frac{q}{2}\right)}\right),
$$

sodass die Reihe (7) in jedem Intervall $0 \leqq r \leqq r_{0}$ gleichmässig konvergiert. Damit finden wir

Lemma 3. Für $r \geqq 0$ und reelle Einheitsvektoren $\xi$ und $\eta$ gilt gleichmässig bezüglich $\xi$ und $\eta$

$$
e^{i r \xi \cdot \eta}=\sum_{n=0}^{\infty} i^{n} N(q ; n) J_{n}(q ; r) P_{n}(q ; \xi \cdot \eta) .
$$

Setzen wir nun $x=r \xi ; x^{\prime}=r^{\prime} \xi^{\prime}$, so wird einerseits

$$
\frac{1}{\omega_{q}} \int_{\Omega_{q}} e^{i x \cdot \eta} \overline{e^{i x^{\prime} \cdot \eta}} d \omega_{q}(\eta)=J_{0}\left(q ;\left|x-x^{\prime}\right|\right) .
$$

Andererseits folgt aus der Parsevalschen Identität, angewandt auf das System der Kugelfunktionen, nach Lemma 3 für die linke Seite der letzten Gleichung

$$
\sum_{n=0}^{\infty} N(q ; n) J_{n}(q ; r) J_{n}\left(q ; r^{\prime}\right) P_{n}\left(q ; \xi \cdot \xi^{\prime}\right)
$$

Damit erhalten wir

Lemma 4. Es sei $x=r \xi ; x^{\prime}=r^{\prime} \xi^{\prime}$. Dann ist

$$
J_{0}\left(q ;\left|x-x^{\prime}\right|\right)=\sum_{n=0}^{\infty} N(q ; n) J_{n}(q ; r) J_{n}\left(q ; r^{\prime}\right) P_{n}\left(q ; \xi \cdot \xi^{\prime}\right) .
$$

Aus der Beziehung

$$
\frac{i^{-n}}{\omega_{q}} \int_{\Omega_{q}} e^{i x \cdot \eta} S_{n}(\eta) d \omega_{q}(\eta)=J_{n}(q ; r) S_{n}(\xi)
$$

ergeben sich viele Integralbeziehungen zwischen Besselfunktionen durch Spezialisierung der Kugelfunktion $S_{n}(\eta)$. Wir wollen als Beispiel hier nur den einfachsten Fall $n=0$ betrachten.

Setzen wir

$$
\begin{array}{ll}
\eta=\eta_{(q)}=t \varepsilon_{q}+\sqrt{1-t^{2}} \eta_{(q-1)}, & \eta_{(q-1)}=\eta^{*}, \\
x=x_{(q)}=x_{q} \varepsilon_{q}+x_{(q-1)}, & x_{(q-1)}=x^{*},
\end{array}
$$

so wird 


$$
J_{0}(q ; r)=\frac{1}{\omega_{q}} \int_{-1}^{+1} \int_{\Omega_{q-1}} e^{i\left(t \cdot x_{q}+\sqrt{1-t^{2}} x^{*} \cdot \eta^{*}\right)}\left(1-t^{2}\right)^{\frac{q-3}{2}} d \omega_{q-1} \wedge d t .
$$

Es ist aber

$$
\int_{\Omega_{q-1}} e^{i \sqrt{1-t^{2}} x^{*} \cdot \eta^{*}} d \omega_{q-1}\left(\eta^{*}\right)=\omega_{q-1} J_{0}\left(q-1 ; \sqrt{1-t^{2}}\left|x_{(q-1)}\right|\right) .
$$

Beachten wir noch, dass

$$
r=\sqrt{x_{q}^{2}+\left|x_{(q-1)}\right|^{2}}
$$

ist, so folgt mit $x_{q}=a ;\left|x_{(q-1)}\right|=b$

Lemma 5. Es gilt

$$
J_{0}\left(q ; \sqrt{a^{2}+b^{2}}\right)=\frac{\omega_{q-1}}{\omega_{q}} \int_{-1}^{+1} e^{i a t} J_{0}\left(q-1 ; \sqrt{1-t^{2}} b\right)\left(1-t^{2}\right)^{\frac{q-3}{2}} d t .
$$

Durch Benutzung weiterer Mittel aus der Theorie der Kugelfunktionen lassen sich alle Besselfunktionen auf die bekannten Besselfunktionen der Dimension $q=2$ elementar zurückführen. Aus (2.40) und (4) folgt nämlich

$$
J_{n}(q ; r)=\left(\frac{r}{2}\right)^{n} \frac{\Gamma\left(\frac{q-1}{2}\right)}{\Gamma\left(n+\frac{q-1}{2}\right)} \frac{\omega_{q-1}}{\omega_{q}} \int_{-1}^{+1} e^{i r t}\left(1-t^{2}\right)^{n+\frac{q-3}{2}} d t
$$

Im klassischen Aufbau der Theorie der Besselfunktionen $J_{r}(*)$, die die in der hier benutzten Schreibweise mit $J_{v}(2 ; r)$ bezeichnet werden müssen, gilt für reelle $v>-1 / 2[7])$

$$
J_{\nu}(r)=J_{\nu}(2 ; r)=\left(\frac{r}{2}\right)^{v} \frac{1}{\sqrt{\pi} \Gamma\left(v+\frac{1}{2}\right)} \int_{-1}^{+1} e^{i r t}\left(1-t^{2}\right)^{1-\frac{1}{2}} d t .
$$

Beachten wir noch, dass

$$
\frac{\omega_{q-1}}{\omega_{q}}=\frac{\Gamma\left(\frac{q}{2}\right)}{\sqrt{\pi} \Gamma\left(\frac{q-1}{2}\right)}
$$


war, so finden wir

$$
J_{n}(q ; r)=\left(\frac{r}{2}\right)^{\frac{2-q}{2}} \Gamma\left(\frac{q}{2}\right) J_{n+\frac{q-2}{2}}(2 ; r),
$$

womit der Anschluss an die üblichen Bezeichnungsweisen hergestellt ist.

\section{§4. Eine Formel der mehrdimensionalen komplexen Integration}

Wir benötigen für die Übertragung der Sommerfeldschen Definition der Hankelfunktionen ein Analogon zur Cauchyschen Integralformel für holomorphe Funktionen mehrerer komplexer Veränderlicher. Es gibt eine Reihe von Integralformeln für mehrfache komplexe Integrale, die unter Ausnutzung der Holomorphie des Integranden mit Hilfe des Kalküls der alternierenden Differentialformen bewiesen werden können [4]. Wir brauchen hier eine spezielle Form, die wir nun ableiten wollen.

In diesem Paragraphen sei stets $q \geqq 3$. Den klassischen Fall $q=\mathbf{2}$ werden wir dann gesondert behandeln und so den Übergang zur bekannten Theorie gewinnen.

Es sei $f\left(z_{1}, z_{2}, \ldots, z_{q}\right)$ eine ganze holomorphe $\left.{ }^{1}\right)$ Funktion der komplexen Veränderlichen $z_{1}, z_{2}, \ldots, z_{q}$. Es sei weiterhin $3_{q}$ ein endlicher Zylinder $(0 \leqq r<R)$

$$
0 \leqq x_{q} \leqq 1 ; \quad r^{2} \leqq x_{1}^{2}+x_{2}^{2}+\cdots+x_{q-1}^{2} \leqq R^{2}
$$

des reellen euklidischen Raumes $E_{q}$. Wir bezeichnen mit $\partial 3_{q}$ seinen orientierten Rand und setzen $\overline{3}_{q}=3_{q} \cup \partial 3_{q}$.

Wir nehmen nun an, dass $q$ komplexwertige Funktionen

$$
z_{j}=z_{j}\left(x_{1}, x_{2}, \ldots, x_{q}\right) ; \quad j=1,2, \ldots, q
$$

in $Z_{q} \cup \partial \bigotimes_{q}$ stetig differenzierbar sind, und für alle $x$ aus $3 q$

$$
z_{1}^{2}+z_{2}^{2}+\cdots+z_{q}^{2}=1
$$

erfüllen. Durch dieses Funktionensystem wird der reelle Zylinder $B_{q}$ auf eine Punktmenge $\mathscr{S}_{q}$ des komplexen Zahlenraumes $C_{q}$ abgebildet. Wegen (3) liegt dabei $\mathfrak{G S}_{q}$ und das Bild $\partial \mathscr{G}_{q}$ ron $\partial 3_{q}$ auf $\Omega_{q}^{*}$.

Wir bilden nun vermittels der Funktionen (2) das Integral

1) Eine ganze holomorphe Funktion ist im ganzen Raum $C_{q}$ holomorph. Die in $\S 4$ formulierten Gesetze gelten auch unter schwächeren Voraussetzungen. Auf die Einzelheiten wird hier verzichtet. 


$$
\int_{\partial \leftrightarrow_{q}} f\left(z_{1}, \ldots, z_{q}\right) d z_{1} \wedge d z_{2} \wedge \ldots \wedge d z_{q-1} .
$$

Dann ist dieses Integral nach dem verallgemeinerten Satz von Stokes gleich

$$
\iint_{\mathscr{S}_{q}} d f \wedge d z_{1} \wedge d z_{2} \wedge \ldots \wedge d z_{q-1} .
$$

Auf Grund der Holomorphie von $f$ ist

$$
d f=\frac{\partial f}{\partial z_{1}} d z_{1}+\frac{\partial f}{\partial z_{2}} d z_{2}+\cdots+\frac{\partial f}{\partial z_{q}} d z_{q},
$$

und es wird aus (5)

$$
\iint_{\Theta_{q}} \frac{\partial f}{\partial z_{q}} d z_{g} \wedge d z_{1} \wedge \ldots \wedge d z_{q-1} .
$$

Aus (3) erhalten wir aber

$$
z_{1} d z_{1}+z_{2} d z_{2}+\cdots+z_{q} d z_{q}=0 .
$$

Damit ist auch

$$
d z_{q} \wedge d z_{1} \wedge \ldots \wedge d z_{q-1}=0
$$

und es verschwindet das Integral (7).

Bezeichnen wir, wie schon in $\S 1$, mit $d \omega_{q}(z)$ die Differentialform (10) $d \omega_{q}(z)=$

$$
\begin{aligned}
z_{1} d z_{2} \wedge d z_{3} \wedge \ldots \wedge d z_{q}-z_{2} d z_{1} \wedge d z_{3} \wedge \ldots \wedge d z_{q}+\cdots \\
+(-1)^{q-1} z_{q} d z_{1} \wedge d z_{2} \wedge \ldots \wedge d z_{q-1}
\end{aligned}
$$

so folgt

$$
\int_{\partial \uplus_{q}} f(z) d \omega_{q}(z)=0,
$$

da sich der Integrand in $q$ Summanden zerlegen lässt, deren Integral jeweils nach dem obigen Ergebnis verschwindet.

Wir erhalten daher

Satz 4. Es sei $\mathscr{G}_{q} \cup \partial \mathscr{G S}_{q}$ das stetig differenzierbare Bild eines abgeschlossenen reellen Zylinders des $E_{q}$, und es liege $\mathfrak{H}_{q} \cup \partial \mathfrak{G S}_{q}$ ganz auf der Punktmenge

$$
\Omega_{q}^{*}: z_{1}^{2}+z_{2}^{2}+\cdots+z_{q}^{2}=1 .
$$


Ist dann $f\left(z_{1}, z_{2}, \ldots, z_{n}\right)$ eine ganze holomorphe Funktion, so gilt mit $f(z)$ $=f\left(z_{1}, z_{2}, \ldots, z_{n}\right)$

$$
\int_{\partial \dot{\omega}_{q}} f(z) d \omega_{q}(z)=0 .
$$

Wir werden diesen Satz benutzen, um eine Übertragung der Formel von Funk und Hecke ins Komplexe zu gewinnen.

Dazu sei von nun an $f(z)$ eine ganze holomorphe Funktion einer Veränderlichen $z$ von der Form $e^{i a z} p(z)$, wobei $p(z)$ ein beliebiges Polynom und $a$ eine positive reelle Konstante ist.

Wir betrachten weiterhin für reelle Einheitsvektoren $\xi^{\prime}$ und positive $R$ die Mannigfaltigkeiten $\Omega_{q}^{(1)}\left(\xi^{\prime} ; R\right)$ und $\Omega_{q}^{(2)}\left(\xi^{\prime} ; R\right)$, die folgendermassen definiert sind:

$$
\begin{aligned}
& \Omega_{q}^{(j)}\left(\xi^{\prime} ; R\right)= \\
& \left\{\eta=\sqrt{1-y^{2}} \xi^{\prime}-y ; \quad y \in E_{q}, y \cdot \xi^{\prime}=0, y^{2} \leqq R^{2},\right. \\
& \operatorname{Im} \sqrt{1-y^{2}} \geqq 0, \operatorname{Re} \sqrt{1-y^{2}}\left\{\begin{array}{l}
\geqq 0 \text { für } j=1 \\
\leqq 0 \text { für } j=2
\end{array}\right\} .
\end{aligned}
$$

Dabei sei auch $R=0$ zugelassen.

Die Punktmengen $\Omega_{q}^{(j)}\left(\xi^{\prime} ; R\right)$ sind reell für $0 \leqq R \leqq 1$. Es ist $\Omega_{q}^{(1)}\left(\xi^{\prime} ; 1\right)$ gleich der Halbsphäre $\eta \cdot \xi^{\prime} \geqq 0$ und $\Omega_{q}^{(2)}\left(\xi^{\prime} ; 1\right)$ gleich der Halbsphäre $\eta \cdot \xi^{\prime} \leqq 0$. Die Orientierung dieser Mannigfaltigkeiten legen wir folgendermassen fest:

Es sei $\varepsilon_{1}^{\prime}, \varepsilon_{2}^{\prime}, \ldots, \varepsilon_{q-1}^{\prime}$ eine orthonormierte Basis des $(q-1)$-dimensionalen linearen Raumes $y \cdot \xi^{\prime}=0$. Dann können wir die Komponenten $y_{\varkappa}^{\prime}=y \cdot \varepsilon_{\varkappa}^{\prime}$ als Parameter zur Darstellung von $\Omega_{q}^{(j)}\left(\xi^{\prime} ; R\right)$ benutzen. Die Basis $\varepsilon_{1}^{\prime}, \varepsilon_{2}^{\prime}, \ldots, \varepsilon_{q-1}^{\prime}$ sei nun so gewählt, dass die Determinante $\left|\varepsilon_{1}^{\prime}, \varepsilon_{2}^{\prime}, \ldots, \varepsilon_{q-1}^{\prime}, \xi^{\prime}\right|_{(q)}=1$ ist. In dem $(q-1)$-dimensionalen Parameterraum setzen wir die kanonische Orientierung voraus.

Dann ist $\Omega_{q}^{(1)}\left(\xi^{\prime} ; 1\right)$ gleich der positiv orientierten Halbsphäre $\eta \cdot \xi^{\prime} \geqq 0$ und $\Omega_{q}^{(2)}\left(\xi^{\prime} ; 1\right)$ gleich der negativ orientierten Halbsphäre $\eta \cdot \xi^{\prime} \leqq 0$.

Für die Dimensionen $q=2$ und $q=3$ lässt sich dies anschaulich erkennen. Im allgemeinen Fall ergibt es sich aus der Berechnung der Differentialform $d \omega_{q}(\eta)$.

Nach (1.11) ist

$$
d \omega_{q}(\eta)=\mid \eta, \eta_{i_{1}}, \eta_{2}, \ldots, \eta_{\mid q-1}{ }_{(q)} d y_{1}^{\prime} \wedge d y_{2}^{\prime} \wedge \ldots \wedge d y_{q-1}^{\prime}
$$

Nun gilt für die Ableitungen $\eta_{\mid \varkappa}$ von $\eta$ nach $y_{*}^{\prime}$ 


$$
\begin{gathered}
\eta_{\mid \varkappa}=-y_{\varkappa}^{\prime}\left[1-\left(y_{1}^{\prime}\right)^{2}-\cdots-\left(y_{q-1}^{\prime}\right)^{2}\right]^{-\frac{1}{2}} \xi^{\prime}-\varepsilon_{\varkappa}^{\prime}, \\
\eta-\sum_{\varkappa=1}^{q-1} y_{\varkappa}^{\prime} \eta_{\mid \varkappa}=\left[1-\left(y_{1}^{\prime}\right)^{2}-\cdots-\left(y_{q-1}^{\prime}\right)^{2}\right]^{-\frac{1}{2}} \xi^{\prime} .
\end{gathered}
$$

Damit ergibt sich nach (13)

$$
\begin{aligned}
d \omega_{g}(\eta) & =(-1)^{q-1}\left|\xi^{\prime}, \varepsilon_{1}^{\prime}, \ldots, \varepsilon_{q-1}^{\prime}\right| \frac{d y_{1}^{\prime} \wedge d y_{2}^{\prime} \wedge \ldots \wedge d y_{q-1}^{\prime}}{\sqrt{1-\left(y_{1}^{\prime}\right)^{2}-\cdots-\left(y_{q-1}^{\prime}\right)^{2}}} \\
& =\frac{d \pi_{q-1}\left(y^{\prime}\right)}{\sqrt{1-\left(y^{\prime}\right)^{2}}},
\end{aligned}
$$

sodass die Orientierung unserer Mannigfaltigkeit durch das Vorzeichen der Wurzel bestimmt wird.

Wir bilden nun mit reellen Vektoren $\xi$ und $\eta$ für $0<R<\infty$

$$
F\left(\xi, \zeta ; \xi^{\prime} ; R\right)=\int_{\Omega_{q}^{(j)}\left(\check{(\Sigma}^{\prime} ; R\right)} f(\xi \cdot \eta) P_{n}(q ; \zeta \cdot \eta) d \omega_{q}(\eta) .
$$

Aus der Definition (12) entnehmen wir für alle orthogonalen Transformationen $A$

$$
A \Omega_{q}^{(j)}\left(\xi^{\prime} ; R\right)=\Omega_{q}^{(j)}\left(A \xi^{\prime} ; R\right) .
$$

Dabei sind die Orientierungen gleich, wenn $\operatorname{det} A=+1$ ist und entgegengesetzt, wenn $\operatorname{det} A=-1$ ist.

Wir erhalten also für orthogonale $A$

$$
\begin{aligned}
& F\left(A \xi, A \zeta ; A \xi^{\prime} ; R\right)=\int_{\Omega_{q}^{(j)}\left(A \xi \xi^{\prime} ; R\right)} f(A \xi \cdot \eta) P_{n}(A \zeta \cdot \eta) d\left(\omega_{q}(\eta)\right. \\
& =\operatorname{det} A \int f(A \xi \cdot \eta) P_{n}(A \zeta \cdot \eta) d \omega_{q}(\eta) \\
& \quad A \Omega_{q}^{(j)}\left(\xi ;_{\left.\xi^{\prime} ; R\right)}\right. \\
& =\operatorname{det} A \int_{\Omega_{q}^{(j)}\left(\xi^{\prime} ; R\right)} f(A \xi \cdot A \eta) P_{n}(A \zeta \cdot A \eta) d \omega_{q}(A \eta)=F\left(\xi, \eta ; \xi^{\prime} ; R\right),
\end{aligned}
$$

sodass für alle orthogonalen $A$

$$
F\left(A \xi, A \zeta ; A \xi^{\prime} ; R\right)=F\left(\xi, \eta ; \xi^{\prime} ; R\right)
$$

gilt.

Da wir zu jedem Vektor $\xi^{\prime}$ eine eigentlich orthogonale Transformation $A$ so finden können, dass $A \xi^{\prime}=\varepsilon_{q}$ ist, genügt es zur Behandlung unserer Integrale, die Mannigfaltigkeiten $\Omega_{q}^{(j)}\left(\varepsilon_{q} ; R\right)$ zu betrachten. Dann ist 


$$
\begin{aligned}
\eta= & \sqrt{1-y_{1}^{2}-y_{2}^{2}-\cdots-y_{q-1}^{2}} \varepsilon_{q}-y_{1} \varepsilon_{1}-y_{2} \varepsilon_{2}-\cdots-y_{q-1} \varepsilon_{q-1} \\
& y_{(q-1)}=\left(y_{1}, \ldots, y_{q-1}\right) \in E_{q-1} ; \quad\left|y_{(q-1)}\right| \leqq R
\end{aligned}
$$

Nach (15) ist

$$
d \omega_{q}(\eta)=\frac{1}{\sqrt{1-y_{(q-1)}^{2}}} d \pi_{q-1}(y) .
$$

Damit wird jetzt ausführlicher

(22) $F\left(\xi, \zeta ; \varepsilon_{q} ; R\right)$

$$
=\int_{\substack{y \in \dot{E}_{q-1} \\ y^{2} \leqq R^{2}}} f\left(\xi \cdot \varepsilon_{q} \sqrt{1-y^{2}}-\xi \cdot y\right) P_{n}\left(\zeta \cdot \varepsilon_{q} \sqrt{1-y^{2}}-\zeta \cdot y\right) \frac{d \pi_{q-1}(y)}{\sqrt{1-y^{2}}} .
$$

Ist, wie vorausgesetzt, $f(z)=e^{i a z} p(z)$, so existiert der Grenzwert

$$
\lim _{R \rightarrow \infty} F\left(\xi, \zeta ; \varepsilon_{q} ; R\right)=F\left(\xi, \zeta ; \varepsilon_{q}\right)
$$

falls $\xi \cdot \varepsilon_{q}>0$ ist.

Setzen wir zur Abkürzung

$$
\Omega_{q}^{(j)}\left(\xi^{\prime}\right)=\Omega_{q}^{(j)}\left(\xi^{\prime} ; \infty\right),
$$

so existiert auf Grund der dargelegten Invarianzeigenschaften

$$
F\left(\xi, \zeta ; \xi^{\prime}\right)=\int_{\Omega_{q}^{(j)}\left(\xi^{\prime}\right)} f(\xi \cdot \eta) P_{n}(\zeta \cdot \eta) d \omega_{q}(\eta)
$$

für alle $\xi, \xi$ und $\xi^{\prime}$ mit $\xi \cdot \xi^{\prime}>0$. Dabei gilt nach (18) für alle orthogonalen $A$

$$
F\left(A \xi, A \zeta ; A \xi^{\prime}\right)=F\left(\xi, \zeta ; \xi^{\prime}\right)
$$

Wir beweisen nun

Satz 5. Es sei mit beliebigem Polynom $p(z)$ und positivem a

$$
f(z)=e^{i a z} p(z) .
$$

$\xi$ und $\zeta$ seien beliebige reelle Einheitsvektoren. Dann erfüllt für alle $\xi$ und $\xi^{\prime \prime}$ mit $\xi \cdot \xi^{\prime}>0 ; \xi \cdot \xi^{\prime \prime}>0$ das durch

$$
F\left(\xi, \zeta ; \xi^{\prime}\right)=\int_{\Omega_{q}^{(j)_{\left(\xi^{\prime}\right)}}} f\left(\xi \cdot \eta_{\eta}\right) P_{n}(\xi \cdot \eta) d \omega_{q}(\eta)
$$

definierte Integral die Beziehung 


$$
F\left(\xi, \zeta ; \xi^{\prime}\right)=F\left(\xi, \zeta ; \xi^{\prime \prime}\right) .
$$

Dieser Satz besagt, dass unser Integral im Halbraum $\xi \cdot \xi^{\prime}>0$ von $\xi^{\prime}$ nicht abhängt. In der Sommerfeldschen Theorie entspricht dies der Verschiebung des Integrationsweges. Wir benötigen hier analog den Satz uiber die komplexe Integration (Satz 4), der diese Gesatzmässigkeit verallgemeinert.

Zum Beweise bemerken wir zunächst, dass es genügt, die Beziehung

$$
F(\xi, \zeta ; \xi)=F\left(\xi, \zeta ; \xi^{\prime}\right)
$$

für $\xi \cdot \xi^{\prime}>0$ zu beweisen. Dann beachten wir, dass wir für $\xi \cdot \xi^{\prime}>0$ durch eine orthogonale Transformation $A$ stets $A \xi=\varepsilon_{q} ; A \xi^{\prime}=$ $\cos \alpha \varepsilon_{q}+\sin \alpha \varepsilon_{q-1}$ mit $0 \leqq \alpha<\pi / 2$ erreichen können.

Wir betrachten nun die reelle Punktmenge

$$
y_{1}^{2}+y_{2}^{2}+\cdots+y_{q-2}^{2}+s^{2} \leqq R^{2}, \quad 0 \leqq \vartheta \leqq \alpha,
$$

die wir als Zylinder $\bar{\S}_{q}$ im euklidischen Raum $E_{q}$ der Veränderlichen $y_{1}, y_{2}, \ldots, y_{q-2}, s, \vartheta$ auffassen können.

Setzen wir weiterhin

$$
\begin{aligned}
z_{k} & =-y_{k} ; \quad k=1,2, \ldots, q-2 ; \\
z_{q-1} & =\sqrt{1-\left(s^{2}+y_{(q-2)}^{2}\right)} \sin \vartheta-s \cos \vartheta ; \\
z_{q} & =\sqrt{1-\left(s^{2}+y_{(q-2)}^{2}\right)} \cos \vartheta+s \sin \vartheta,
\end{aligned}
$$

so stellt (mit $\operatorname{Im} \sqrt{1-\left(s^{2}+y_{(q-2)}^{2}\right)} \geqq 0$ ) für konstante $\vartheta$

$$
\eta_{(q)}=\left(z_{1}, z_{2}, \ldots, z_{q}\right)
$$

je nach der Festlegung des Vorzeichens der Wurzel für positive Radikanden, die Punktmengen

$$
\Omega_{q}^{(j)}\left(\cos \vartheta \varepsilon_{q}+\sin \vartheta \varepsilon_{q-1} ; R\right)
$$

mit $j=1$ oder $j=2$ dar.

Für alle $\vartheta$ folgt aus (28) und (29) $\eta_{(q)}^{2}=1$. Vermittels (28) wird also der in (27) definierte Zylinder $\beta_{q}$ auf eine Punktmenge $\mathfrak{G}_{q}$ abgebildet, die ganz auf der komplexen Menge $\Omega_{q}^{*}$ liegt. Die durch (28) vermittelte Abbildung ist stetig und für $s^{2}+y_{1}^{2}+\cdots+y_{q-2}^{2} \neq 1$ auch stetig differenzierbar.

Es kann das Polynom $P_{n}(\zeta \cdot \eta)$ auf $\Omega_{q}^{*}$ als homogenes Polynom vom Grade $n$ in den Variablen $\eta_{1}, \eta_{2}, \ldots, \eta_{g}$ geschrieben werden. Der Integrand des in Satz 5 genannten Integrals lässt sich daher als ganze holomorphe Funktion der komplexen Veränderlichen $\eta_{1}, \eta_{2}, \ldots, \eta_{q}$ schreiben. 
Die wesentlichen Voraussetzungen des Satzes 4 sind damit erfüllt. Die störenden Singularitäten der Abbildungsfunktionen (28) in der Nähe der Parameterwerte $s^{2}+y_{1}^{2}+\cdots+y_{q-2}^{2}=1$ werden sich bei den nun folgenden Berechnungen als integrierbar erweisen, sodass sie die Gesamtstruktur nicht stören.

Für $R<1$ treten diese Schwierigkeiten noch nicht auf. Für $R>1$ müssten wir Satz 4 zunächst auf die Bilder der Zylinder $0 \leqq \vartheta \leqq \alpha$; $0 \leqq s^{2}+y_{1}^{2}+\cdots+y_{q-2}^{2} \leqq 1-\delta \quad$ und $\quad 0 \leqq \vartheta \leqq \alpha ; \quad 1+\delta \leqq s^{2}+y_{1}^{2}+$ $\cdots+y_{q-2}^{2} \leqq R^{2}$ anwenden und dann durch den Grenzübergang $\delta \rightarrow+0$ den Anschluss herstellen. Auf die Einzelheiten, die sich aus den nun folgenden Rechnungen leicht ablesen lassen, werde hier verzichtet.

Das Bild $\partial \mathscr{G}_{q}$ des Zylinderrandes $\partial g_{q}$ besteht aus den Bildern

$$
\Omega_{q}^{(j)}\left(\varepsilon_{q} ; R\right) \quad \text { und } \quad \Omega_{q}^{(j)}\left(\cos \alpha \varepsilon_{q}+\sin \alpha \varepsilon_{q-1} ; R\right)
$$

der ebenen Brandungen $\vartheta=0$ und $\vartheta=\alpha$ des Zylinders sowie dem Bild des Mantels

$$
y_{1}^{2}+y_{2}^{2}+\cdots+y_{q-2}^{2}+s^{2}=R^{2}, \quad 0 \leqq \vartheta \leqq \alpha,
$$

das wir mit $\Gamma(\alpha ; R)$ bezeichnen wollen. Nach Satz 4 wird damit für $\xi^{\prime}=\cos x \varepsilon_{q}+\sin \alpha \varepsilon_{q-1}$

$$
\begin{aligned}
& \int_{\Omega_{q}^{\left.(j) \varepsilon_{q} ; R\right)}} f\left(\varepsilon_{q} \cdot \eta\right) P_{n}(\zeta \cdot \eta) d \omega_{q}(\eta)-\int_{\Omega_{q}^{(j)}\left(\xi^{\prime} ; R\right)} f\left(\varepsilon_{q} \cdot \eta\right) P_{n}(\zeta \cdot \eta) d \omega_{q}(\eta) \\
& =\int_{\Gamma(\alpha ; R} f\left(\varepsilon_{q} \cdot \eta\right) P_{n}(\zeta \cdot \eta) d \omega_{q}(\eta) .
\end{aligned}
$$

Zum Beweise von Satz 5 müssen wir daher nun zeigen, dass das letzte Integral für $R \rightarrow \infty$ verschwindet.

Zur Parametrisierung der Mannigfaltigkeit $\Gamma(x ; R)$ setzen wir

$$
y_{(q-2)}=\left(y_{1}, y_{2}, \ldots, y_{q-2}\right)=\sqrt{R^{2}-s^{2}} \eta_{(q-2)} ; \quad \eta_{(q-2)} \in \Omega_{q-2} .
$$

Damn wird aus (28) und (29) mit (32)

$$
\begin{aligned}
\eta & \\
\left(\sqrt{1-R^{2}} \cos \vartheta+s \sin \vartheta\right) \varepsilon_{q}+\left(\sqrt{1-R^{2}} \sin \vartheta\right. & -s \cos \vartheta) \varepsilon_{q-1} \\
& -\sqrt{R^{2}-s^{2}} \eta_{(q-2)} .
\end{aligned}
$$

Nun ist nach (1.13)

$$
\begin{aligned}
d \omega_{q}(\eta) & =d \omega_{q-1} \wedge d \eta_{q}+(-1)^{q-1} \eta_{q q} d \pi_{q-1} \\
& =d \omega_{q-2} \wedge d \eta_{q-1} \wedge d \eta_{q}+(-1)^{q} d \pi_{q-2} \wedge\left(\eta_{q-1} d \eta_{q}-\eta_{q} d \eta_{q-1}\right)
\end{aligned}
$$


Nach (1.16) ist

$$
d \omega_{q-2}\left(\sqrt{R^{2}-s^{2}} \eta_{(q-2)}\right)=\left(R^{2}-s^{2}\right)^{\frac{q-2}{2}} d \omega_{q-2}(\eta)
$$

und nach (1.19) ist

$$
d \pi_{q-2}\left(\sqrt{R^{2}-s^{2}} \eta\right)=-\left(R^{2}-s^{2}\right)^{\frac{q-4}{2}} s d s \wedge d \omega_{q-2}(\eta) .
$$

Es ist weiterhin

$$
\begin{gathered}
d \eta_{q-1}=\eta_{q} d \vartheta-\cos \vartheta d s, \\
d \eta_{q}=-\eta_{q-1} d \vartheta+\sin \vartheta d s
\end{gathered}
$$

und somit

$$
\begin{gathered}
d \eta_{q-1} \wedge d \eta_{q}=\left(\eta_{q} \sin \vartheta-\eta_{q-1} \cos \vartheta\right) d \vartheta \wedge d s=s d \vartheta \wedge d s \\
\eta_{q-1} d \eta_{q}-\eta_{q} d \eta_{q-1}=-\left(1-\left(R^{2}-s^{2}\right)\right) d \vartheta+\sqrt{1-R^{2}} d s
\end{gathered}
$$

Durch Zusammenfassung wird daher auf $\Gamma(\alpha ; R)$

$$
d \omega_{q}(\eta)=\left(R^{2}-s^{2}\right)^{\frac{q-4}{2}} s d \omega_{q-2} \wedge d \vartheta \wedge d s .
$$

Damit erhalten wir

$$
\begin{gathered}
\int_{I^{\prime}(\alpha ; R)} f\left(\varepsilon_{q} \cdot \eta\right) P_{n}(\zeta \cdot \eta) d \omega_{q}(\eta) \\
=\int_{-R}^{R} \int_{0}^{\alpha}\left(\int_{\Omega_{q-2}}^{\alpha} f\left(\sqrt{1-R^{2}} \cos \vartheta+s \sin \vartheta\right) p_{n}\left(s, \vartheta, \eta_{(q-2)}\right) d()_{q-2}(\eta)\right) d \vartheta d s . \\
p_{n}\left(s, \vartheta, \eta_{(q-2)}\right)=P_{n}(\zeta \cdot \eta) .
\end{gathered}
$$

Nun gibt es für $R \geqq R_{0}>1$ eine ganze Zahl $N$ so, dass gleichmässig in $0 \leqq s \leqq R$

$$
\left|f\left(\sqrt{1-R^{2}} \cos \vartheta+s \sin \vartheta\right)\right|=O\left(e^{-a \sqrt{R^{2}-1} \cos \vartheta} R^{x}\right)
$$

gilt. Da andererseits $P_{n}(\zeta \cdot \eta)$ nur wie $R^{n}$ wachsen kann, folgt durch eine Gesamtabschätzung, dass das Integral (42) für $R \rightarrow \infty$ verschwindet. Damit ist Satz 5 bewiesen.

Zur weiteren Berechnung unseres Integrals (25) können wir daher $\xi=\xi^{\prime}$ setzen und erhalten wegen der Orthogonalinvarianz 


$$
F(\xi, \zeta ; \xi)=\Phi(\xi \cdot \zeta)
$$

Als Funkticn von $\zeta$ ist $F(\xi, \zeta ; \xi)$ eine Kugelfunktion der Ordnung $n$. Es folgt also

$$
F(\xi, \zeta ; \xi)=\mu_{n}^{(j)} P_{n}(\xi \cdot \zeta) .
$$

Zur Berechnung der Konstanten $\mu_{n}^{(j)}$ setzen wir nun $\xi=\zeta=\varepsilon_{q}$ und erhalten

$$
\mu_{n}^{(j)}=\int_{\left.\Omega_{q}^{(j)}\right)_{\left(\varepsilon_{q}\right)}} f\left(\eta \cdot \varepsilon_{q}\right) P_{n}\left(\eta \cdot \varepsilon_{q}\right) d \omega_{q}(\eta)
$$

Die Parameterdarstellung (20) liefert uns dann wegen (21)

$$
\mu_{n}^{(j)}=\int_{y \in E_{q-1}} f\left(\sqrt{1-y^{2}}\right) P_{n}\left(\sqrt{1-y^{2}}\right) \frac{d \pi_{q-1}(y)}{\sqrt{1-y^{2}}},
$$

wobei sich die Fäile $j=1$ und $j=2$ durch

$$
\begin{aligned}
& \operatorname{Re}\left(\sqrt{1-y^{2}}\right) \geqq 0 \quad \text { für } j=1, \\
& \operatorname{Re}\left(\sqrt{1-y^{2}}\right) \leqq 0 \text { für } j=2
\end{aligned}
$$

unterscheiden. Führen wir in $E_{q-1}$ Polarkoordinaten

$$
y_{(q-1)}=r \eta_{(q-1)} ; \quad 0 \leqq r ; \eta_{(q-1)} \in \Omega_{q-1} ;
$$

ein, so folgt

$$
\mu_{n}^{(j)}=\omega_{q-1} \int_{0}^{\infty} f\left(\sqrt{1-r^{2}}\right) P_{n}\left(\sqrt{1-r^{2}}\right) \frac{r^{q-2} d r}{\sqrt{1-r^{2}}} .
$$

Setzen wir num $t=\sqrt{1-r^{2}}$, so ergeben sich für $j=1$ und $j=2$ die skizzierten. Integrationswege. Eine übliche Deformation dieser Wege führt dann auf

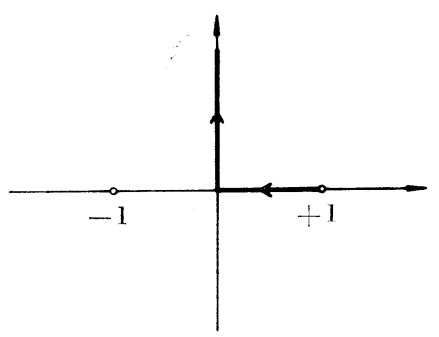

$j=1$

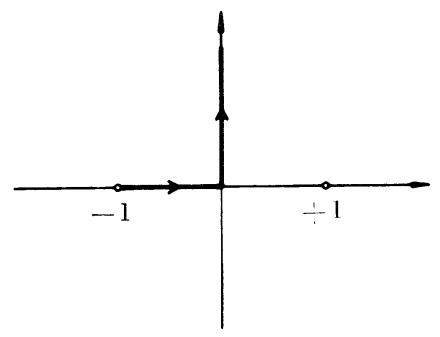

$j=2$ 


$$
\begin{aligned}
\mu_{n}^{(1)}= & -\omega_{q-1} \int_{1+0 i}^{1+\infty i} f(t) P_{n}(t)\left(1-t^{2}\right)^{\frac{q-3}{2}} d t, \\
& -1+\infty i \\
\mu_{n}^{(2)}= & -\omega_{q-1} \int_{-1+0 i}^{\infty} f(t) P_{n}(t)\left(1-t^{2}\right)^{\frac{q-3}{2}} d t .
\end{aligned}
$$

Wir können damit unser Ergebnis zusammenfassen und erhalten

Lemma 6. Es seien $\xi$, $\xi^{\prime}$ reelle Einheitsvektoren und es sei

$$
f(z)=e^{i a z} p(z)
$$

mit einer positiven Konstanten a und einem Polynom $p(z)$. Dann ist für $\xi \cdot \xi^{\prime}>0$ und beliebige reelle $\zeta \in \Omega_{q}$

$$
\int_{\left.\Omega_{q}^{(j)}\right)_{\left(\xi^{\prime}\right)}} f(\xi \cdot \eta) P_{n}(q ; \zeta \cdot \eta) d \omega_{q}(\eta)=\mu_{n}^{(j)} P_{n}(q ; \xi \cdot \zeta)
$$

mit

$$
\begin{aligned}
\mu_{n}^{(1)}= & -\omega_{q-1} \int_{1+0 i}^{1+\infty i} f(t) P_{n}(q ; t)\left(1-t^{2}\right)^{\frac{q-3}{2}} d t, \\
\mu_{n}^{(2)}= & -\omega_{q-1} \int_{-1+0 i}^{-1+\infty i} f(t) P_{n}(q ; t)\left(1-t^{2}\right)^{\frac{q-3}{2}} d t .
\end{aligned}
$$

Multiplizieren wir beide Seiten dieser Identität mit einer Kugelfunktion $\left(N / \omega_{q}\right) S_{n}(\zeta)$ und integrieren über $\Omega_{q}$ bezüglich $\zeta$, so folgt aus dem Additionstheorem der Kugelfunktionen

Satz 6. Unter den Voraussetzungen von Lemma 6 ist für jede Kugelfunktion $\mathrm{S}_{n}(\eta)$

$$
\int_{\Omega_{q}^{(j)}} f(\xi \cdot \eta) S_{n}(\eta) d \omega_{q}(\eta)=\mu_{n}^{(j)} S_{n}(\xi),
$$

wobei die Koeffizienten $\mu_{n}^{(j)}$ in Lemma 6 gegeben sind.

Wir wollen nun noch eine weitere Integrationsmannigfaltigkeit untersuchen und betrachten dazu die komplexe Punktmenge

$$
\begin{aligned}
& \Sigma_{q}\left(\xi^{\prime} ; R\right)= \\
& \left\{\eta=\sqrt{1+y^{2}} \xi^{\prime}+i y ; y \in E_{q} ;|y| \leqq R ; y \cdot \xi^{\prime}=0\right\} .
\end{aligned}
$$


Die Orientierung denken wir uns wie bei $\Omega_{q}^{(1)}\left(\xi^{\prime} ; R\right)$ festgelegt. Wir setzen $\Sigma_{q}\left(\xi^{\prime} ; \infty\right)=\Sigma_{q}\left(\xi^{\prime}\right)$.

Es sei nun mit einer positiven Konstanten $a$ und einem Polynom $p(z)$

$$
f(z)=e^{-a z} p(z) \text {. }
$$

Setzen wir dann

$$
F\left(\xi, \zeta ; \xi^{\prime} ; R\right)=\int_{\Sigma_{q}\left(\xi^{\prime} ; R\right)} f(\xi \cdot \eta) P_{n}(\zeta \cdot \eta) d \omega_{q}(\eta),
$$

so gilt wieder für alle orthogonalen $A$

$$
F\left(A \xi, A \zeta ; A \xi^{\prime} ; R\right)=F\left(\xi, \zeta ; \xi^{\prime} ; R\right),
$$

und wir können uns zur Untersuchung der analytischen Eigenschaften dieses Integrals auf den Fall $\xi^{\prime}=\varepsilon_{q}$ beschränken.

Die Punktmenge $\Sigma_{q}\left(\varepsilon_{q} ; R\right)$ geht aus $\Omega_{q}^{(1)}\left(\varepsilon_{q} ; R\right)$ nach (20) hervor, wenn wir für $x=1,2, \ldots, q-1$ jeweils $y_{x}$ durch $-i y_{*}$ ersetzen. Dann wird für die Parameterdarstellung (51)

$$
d \omega_{q}(\eta)=(-i)^{q-1} \frac{d \pi_{q-1}(y)}{\sqrt{1+y^{2}}} .
$$

Ist nun $\xi \cdot \varepsilon_{q}>0$, so existiert offenbar der Grenzwert

$$
F\left(\xi, \zeta ; \varepsilon_{q}\right)=F\left(\xi, \zeta ; \varepsilon_{q} ; \infty\right) .
$$

Analog zum Beweise von Satz 5 können wir nun auch

$$
F\left(\varepsilon_{q}, \zeta ; \varepsilon_{q}\right)=F\left(\cos x \varepsilon_{q}+\sin x \varepsilon_{q-1}, \zeta ; \varepsilon_{q}\right)
$$

für $0 \leqq \alpha<\pi / 2$ zeigen. Die Einzelheiten seien hier übergangen.

Damit gilt dann für alle $\xi$, $\xi^{\prime}$ mit $\xi \cdot \xi^{\prime}>0$ und beliebige reelle $\zeta \in \Omega_{q}$

$$
F(\xi, \zeta ; \xi)=F\left(\xi, \zeta ; \xi^{\prime}\right),
$$

und es folgt aus der Orthogonalinvarianz wiederum, dass $F(\xi, ; \xi \xi)$ nur von Skalarprodukt $\xi \cdot \zeta$ abhängt. Es wird also auch

$$
F(\xi, \zeta ; \xi)=\sigma_{n} P_{n}(\xi \cdot \zeta),
$$

wobei wir zur Bestimmung von $\sigma_{n}$ wieder $\xi=\zeta=\varepsilon_{q}$ setzen und dann

$$
\begin{aligned}
\sigma_{n} & =\int_{\Sigma_{\left.q^{(} \varepsilon_{q}\right)}} f\left(\varepsilon_{q} \cdot \eta\right) P_{n}\left(\varepsilon_{q} \cdot \eta\right) d \omega_{q}(\eta) \\
& =i^{1-q} \int_{E_{q-1}} f\left(\sqrt{1+y^{2}}\right) P_{n}\left(\sqrt{1+y^{2}}\right) \frac{d \pi_{q-1}(y)}{\sqrt{1+y^{2}}}
\end{aligned}
$$


erhalten. Die Ausrechnung liefert uns dann

$$
\sigma_{n}=i^{1-q} \omega_{q-1} \int_{1}^{\infty} f(t) P_{n}(t)\left(t^{2}-1\right)^{\frac{q-3}{2}} d t .
$$

Damit ergibt sich dann zunächst

Lemma 7. Es sei $\xi^{\prime}$ ein beliebiger reeller Einheitsvektor und

$$
f(z)=e^{-a z} p(z)
$$

mit einem Polynom $p(z)$ und einer Konstanten $a>0$. Dann ist für $\xi \cdot \xi^{\prime}>0$ und beliebige $\zeta \in \Omega_{q}$

$$
\int_{\check{\sim}_{q}\left(\xi^{\prime}\right)} f(\xi \cdot \eta) P_{n}(q ; \zeta \cdot \eta) d \omega_{q}(\eta)=\sigma_{n} P_{n}(q ; \xi \cdot \zeta)
$$

mit

$$
\sigma_{n}=i^{1-q} \omega_{q-1} \int_{1}^{\infty} f(t) P_{n}(q ; t)\left(t^{2}-1\right)^{\frac{q-3}{2}} d t .
$$

Durch Multiplikation mit $\left(N / \omega_{q}\right) S_{n}(\eta)$ und nachfolgende Integration über $\Omega_{q}$ erhalten wir dann

Satz 7. Es sei $S_{n}(\eta)$ eine Kugelfunktion der Ordnung $n$ und Dimension $q$. Dann ist für $\xi \cdot \xi^{\prime}>0$

$$
\int_{\Sigma_{q}\left(\xi^{\prime}\right)} f(\xi \cdot \eta) S_{n}(\eta) d \omega_{q}(\eta)=\sigma_{n} S_{n}(\xi),
$$

wenn $f(z)$ und $\sigma_{n}$ die in Lemma 7 genannte Bedeutung haben.

\section{§ 5. Die Sommerfeldschen Integrale}

Wir haben unsere Betrachtungen im vorigen Paragraphen auf die Dimensionen $q \geqq 3$ beschränkt. Der Fall $q=2$ spielt eine besondere Rolle, da hier die Methoden der klassischen Funktionentheorie unmittelbar anwendbar sind, wie Sommerfeld in seinen bekannten Arbeiten um die Jahrhundertwende gezeigt hat.

Wir wollen diese Theorie nun hier in den allgemeinen Zugang einschliessen. Dazu müssen unsere Bezeichnungsweisen zunächst in die klassischen Formulierungen übersetzt werden.

Wir beginnen mit den Punktmengen $\Omega_{2}, \Omega_{2}^{(j)}\left(\xi^{\prime}\right)$ und $\Sigma_{2}\left(\xi^{\prime}\right)$ sowie den Kugelfunktioner. 
Es ist $\Omega_{2}$ der Einheitskreis, dessen Punkte durch

$$
\xi_{1}=-\sin \varphi ; \quad \xi_{2}=\cos \varphi ; \quad 0 \leqq \varphi \leqq 2 \pi ;
$$

parametrisiert werden können. Hier wird

$$
d \omega_{2}=\xi_{1} d \xi_{2}-\xi_{2} d \xi_{1}=d \varphi .
$$

Die Funktionen $\left(x_{2}-i x_{1}\right)^{n}$ und $\left(x_{2}+i x_{1}\right)^{n}$ sind homogen vom Grade $n$ und harmonisch. Mit der Parameterdarstellung (1) werden Kugelfunktionen der Ordnung $n$ durch $e^{i n \varphi}$ und $e^{-i n \varphi}$ erhalten. Es gibt zwei linear unabhängige Kugelfunktionen zu jeder Ordnung $n \geqq 1$ und es bilden die Funktionen $S_{0}(\xi)=1 / \sqrt{2 \pi}$,

(3) $\quad S_{n, 1}(\xi)=\frac{1}{\sqrt{\pi}} \cos n \varphi ; \quad S_{n, 2}(\xi)=\frac{1}{\sqrt{\pi}} \sin n \varphi ; \quad n=1,2, \ldots$,

ein Orthonormalsystem.

Setzen wir

$$
\eta_{1}=-\sin \psi ; \quad \eta_{2}=\cos \psi
$$

so wird einerseits

$$
S_{n, 1}(\xi) S_{n, 1}(\eta)+S_{n, 2}(\xi) S_{n, 2}(\eta)=\frac{1}{\pi} \cos n(\varphi-\psi)
$$

Andererseits ist

$$
\xi \cdot \eta=\cos (\varphi-\psi) .
$$

Nach dem Additionstheorem (Satz 1) ist die rechte Seite von (5) gleich

$$
\frac{N(2 ; n)}{\omega_{2}} P_{n}(2 ; \xi \cdot \eta)=\frac{1}{\pi} P_{n}(2 ; \xi \cdot \eta)
$$

Wir erhalten daher

$$
P_{n}(2 ; t)=\cos n(\arccos t)
$$

Setzen wir nun weiterhin

$$
\xi_{1}^{\prime}=-\sin \varphi^{\prime} ; \quad \xi_{2}^{\prime}=\cos \varphi^{\prime},
$$

so werden die Punktmengen $\Omega_{2}^{(j)}\left(\xi^{\prime}\right)$ durch

$$
\begin{aligned}
& \eta_{1}=-\sqrt{1-y^{2}} \sin \varphi^{\prime}-y \cos \varphi^{\prime} ; \quad-\infty<y<\infty, \\
& \eta_{2}=+\sqrt{1-y^{2}} \cos \varphi^{\prime}-y \sin \varphi^{\prime}
\end{aligned}
$$

parametrisiert, wobei wir die Vorzeichen der Wurzel noch festlegen müssen. 

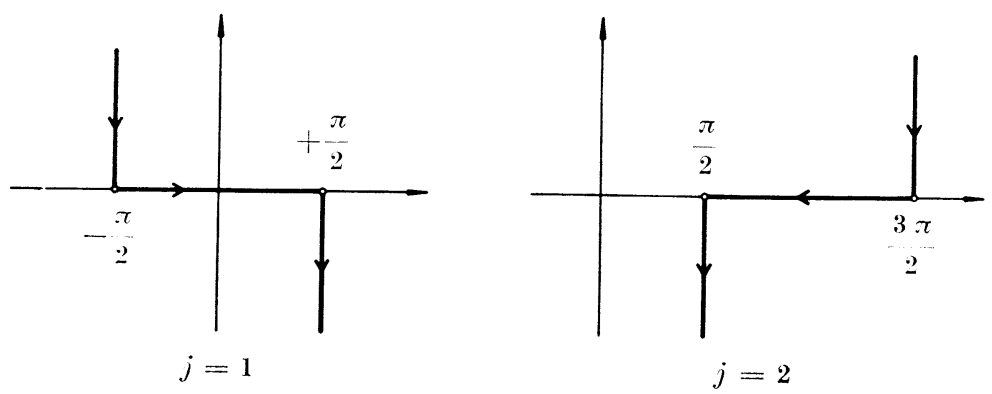

Setzen wir nun $y=\sin \alpha$ und $\sqrt{1-y^{2}}=\cos \alpha$, so erhalten wir die Vorzeichenfestlegungen der Mannigfaltigkeiten $\Omega_{2}^{(1)}$ und $\Omega_{2}^{(2)}$, wenn $\alpha$ die skizzierten Wege in der komplexen Zahlenebene durchläuft. Mit der Parametrisierung $y=\sin \alpha$ wird also

$$
\eta_{1}=-\sin \left(\varphi^{\prime}+\alpha\right) ; \quad \eta_{2}=\cos \left(\varphi^{\prime}+\alpha\right) .
$$

Setzen wir $S_{n}(\eta)=\left(\eta_{2}-i \eta_{1}\right)^{n}$, so folgt auf $\Omega_{2}^{(j)}\left(\xi^{\prime}\right)$

$$
S_{n}(\eta)=e^{i n\left(\alpha+\varphi^{\prime}\right)} .
$$

Weiterhin ergibt sich sofort

$$
d \omega_{2}(\eta)=d \alpha .
$$

Zur Interpretation des Satzes 6 setzen wir der Einfachheit halber $f(z)=e^{i r z}$. Dann wird

$$
\int_{\Omega_{2}^{(1)}\left(\Sigma^{\prime}\right)} e^{i r \xi \eta \eta} S_{n}(\eta) d \omega_{2}(\eta)=\int_{-\frac{\pi}{2}+\infty i}^{\frac{\pi}{2}-\infty i} e^{i r \cos \left(\alpha+\varphi^{\prime}-\varphi\right)} e^{i n\left(\alpha+\varphi^{\prime}\right)} d x .
$$

Die Bedingung $\xi \cdot \xi^{\prime}>0$ bedeutet hier $\left|\varphi-\varphi^{\prime}\right|<\pi / 2$. Setzen wir nun noch $\beta=\alpha+\varphi^{\prime}$, so erhalten wir das Sommerfeldsche Integral

$$
\int_{\Omega_{2}^{(1)}\left(\xi^{\prime}\right)} e^{i r \xi \cdot \eta} S_{n}(\eta) d \omega_{2}(\eta)=\int_{-\frac{\pi}{2}+\varphi^{\prime}+\infty i} e^{i r \cos (\beta-\psi)} e^{i n \beta} d \beta .
$$

Die Unabhängigkeit von $\varphi^{\prime}$ für $\left|\varphi^{\prime}-\varphi\right|<\pi / 2$, und damit vom Vektor $\xi^{\prime}$ für $\xi \cdot \xi^{\prime}>0$ drückt sich hier durch die Möglichkeit der Verschiebung des Integrationsweges aus.

Analog erhalten wir 
(16)

$$
\int_{\Omega_{2}^{(2)}\left(\xi^{\prime}\right)} e^{i r \xi \cdot \eta} S_{n}(\eta) d \omega_{2}(\eta)=\int_{\frac{3 \pi}{2}+\eta^{\prime}+\infty i} e^{i r \cos (\beta-\varphi)} e^{i n \beta} d \beta .
$$

Die rechten Seiten von (15) und (16) können wir nun umformen indem wir zunächst $\varphi^{\prime}=\varphi$ setzen und dann $\gamma=\beta-\varphi$ als neue Integrationsveränderliche einführen. Damit wird aus (15)

$$
\begin{gathered}
\int_{\Omega_{2}^{(1)}\left(\xi^{\prime}\right)} e^{i r \xi \eta \eta} S_{n}(\eta) d \omega_{2}(\eta)=e^{i n \varphi} \int_{-\frac{\pi}{2}+\infty i}^{\frac{\pi}{2}-\infty i} e^{i r \cos \gamma} e^{i n \gamma} d \gamma \\
=e^{i n \varphi} 2 \int_{0}^{\frac{\pi}{2}-\infty i} e^{i r \cos \gamma} \cos n \gamma d \gamma \\
=-2 e^{i n \varphi} \int_{1+0 i}^{\infty+\infty i} e^{i r t} P_{n}(2 ; t) \frac{d t}{\sqrt{1-t^{2}}}
\end{gathered}
$$

wobei die letzte Umformung durch die Substitution $t=\cos \gamma$ und anschliessende Deformation des Integrationsweges erfolgt.

Schreiben wir dieses Ergebnis in der Form

$$
\begin{gathered}
\int_{\left.\Omega_{2}^{(j)}\right)_{(\xi)}} e^{i r \xi \eta \eta} S_{n}(\eta) d \omega_{2}(\eta)=\mu_{n}^{(1)} S_{n}(\xi), \\
\mu_{n}^{(1)}=-2 \int_{1+0 i}^{1+\infty i} e^{i r t} P_{n}(2 ; t) \frac{d t}{\sqrt{1-t^{2}}},
\end{gathered}
$$

so ist Satz 6 für $j=1$ und $f(z)=e^{i r z}$ im Falle $q=2$ bewiesen. Für $j=2$ erhalten wir ein analoges Ergebnis mit

$$
\mu_{n}^{(2)}=-2 \int_{-1}^{-1+\infty i} e^{i r t} P_{n}(2 ; t) \frac{d t}{\sqrt{1-t^{2}}} .
$$

Von Normierungsfaktoren abgesehen liefern diese Integrale bekanntlich die Sommerfeldschen Darstellungen der Hankelfunktionen erster und zweiter Art.

Wir wollen nun abschliessend noch die Integrale über $\Sigma_{2}\left(\xi^{\prime}\right)$ diskutieren. Die Mannigfaltigkeit $\quad \Sigma_{2}\left(\xi^{\prime}\right)$ wurde durch 


$$
\eta_{1}=-\sqrt{1+y^{2}} \sin \varphi^{\prime}+i y \cos \varphi^{\prime}
$$

$$
\eta_{2}=\sqrt{1+y^{2}} \cos \varphi^{\prime}+i y \sin \varphi^{\prime} \quad-\infty<y<\infty ;
$$

parametrisiert. Setzen wir $y=\sinh t ; \sqrt{1+y^{2}}=\cosh t$, so folgt

$$
\begin{aligned}
& \eta_{1}=-\sin \left(\varphi^{\prime}-i t\right), \\
& \eta_{2}=\cos \left(\varphi^{\prime}-i t\right)
\end{aligned}
$$

und

$$
d \omega_{2}(\eta)=-i d t
$$

sowie

$$
S_{n}(\eta)=e^{i n\left(\phi^{\prime}-i t\right)}
$$

Die Funktion $f(z)$ sei $e^{-r z}$. Dann ist für $\left|\varphi-\varphi^{\prime}\right|<\pi / 2$

$$
\begin{aligned}
& \int_{\Sigma_{2}\left(\xi^{\prime}\right)} e^{-r \xi \cdot \eta} S_{n}(\eta) d \omega_{2}(\eta)=-i \int_{-\infty}^{+\infty} e^{-r \cos \left(\varphi^{\prime}-\varphi-i t\right)} e^{i n\left(\varphi^{\prime}-i t\right)} d t \\
& =\int_{\varphi^{\prime}+i \infty}^{\gamma^{\prime}-i \infty} e^{-r \cos (\beta-\varphi)} e^{i n \beta} d \beta=e^{i n \varphi} \int_{i \infty}^{-i \infty} e^{-r \cos \beta} e^{i n \beta} d \beta \\
& =e^{i n \varphi} i \int_{+\infty}^{-\infty} e^{-r \cosh s} e^{-n s} d s .
\end{aligned}
$$

Dies können wir durch die Substitution $t=\cosh s$ umformen zu

$$
-2 i e^{i n \varphi} \int_{1}^{\infty} \frac{e^{-r t}}{\sqrt{t^{2}-1}} P_{n}(2 ; t) d t=\sigma_{n} e^{i n q}
$$

mit

$$
\sigma_{n}=-2 i \int_{1}^{\infty} \frac{e^{-r t}}{\sqrt{t^{2}-1}} P_{n}(2 ; t) d t .
$$

Dieses Ergebnis entspricht Satz 7 für $q=2$ und $f(z)=e^{-r z}$. Durch

$$
\int_{\Omega_{2}^{(j)}\left(\xi^{\prime}\right)} e^{i x \cdot \eta} S_{n}(\eta) d \omega_{2}(\eta) \text { und } \int_{\Sigma_{2}\left(\xi^{\prime}\right)} e^{-x \cdot \eta} S_{n}(\eta) d \omega_{2}(\eta)
$$


werden nun jeweils in den Halbräumen $x \cdot \xi^{\prime}>0$ analytische Funktionen von $x=\left(x_{1}, x_{2}\right)$ definiert. Bei festem $\xi^{\prime}$ können wir unter dem Integral nach den Komponenten von $x$ differenzieren. Wegen $\eta^{2}=1$ stellen diese Funktionen dann Lösungen von $\Delta U+U=0$ bezw. $\Delta U-U=0$ dar.

Die Theorie der voraufgegangenen Paragraphen liefert somit analytische Darstellungen der Lösungen dieser Differentialgleichungen, die nach dem Prinzip der analytischen Fortsetzung auf den ganzen Raum $x^{2}>0$ eindeutig ausgedehnt werden können.

Im nächsten Abschnitt wollen wir diesen Gedanken auf beliebige Dimensionen übertragen und die entsprechenden Hankel- und Kelvinfunktionen definieren.

\section{§6. Die mehrdimensionalen Hankel- und Kelvinfunktionen}

Nach Satz 6 existiert für $x=r \xi$ und $\xi \cdot \xi^{\prime}>0$

$$
U_{n}^{(j)}(x)=(-1)^{j-1} \frac{2 i^{-n}}{\omega_{q}} \int_{\Omega_{q}^{(j)}} e^{i x \cdot \eta} S_{n}(\eta) d \omega_{q}(\eta) .
$$

Bei festem $\xi^{\prime}$ können wir bezüglich $x$ differenzieren und erhalten wegen $\eta^{2}=1$

$$
\Delta U_{n}^{(j)}+U_{n}^{(j)}=0
$$

Andererseits ist nach Satz 6

$$
U_{n}^{(j)}(x)=H_{n}^{(j)}(q ; r) S_{n}(\xi)
$$

mit

$$
H_{n}^{(1)}(q ; r)=-\frac{i^{-n} 2 \omega_{q-1}}{\omega_{q}} \int_{1-0 i}^{1-\infty_{i}} e^{i r t} P_{n}(q ; t)\left(1-t^{2}\right)^{\frac{q-3}{2}} d t
$$

und

$$
H_{n}^{(2)}(q ; r)=\frac{i^{-n} 2 \omega_{q-1}}{\omega_{q}} \int_{-1-0 i}^{\infty i} e^{i r t} P_{n}(q: t)\left(1-t^{2}\right)^{\frac{q-3}{2}} d t
$$

Beachtet man, dass

$$
P_{n}(2 ; t)=\cos n(\arccos t)
$$

ist, und $\omega_{1}=2$ gesetzt wird, so stimmt diese Integraldarstellung für $q=2$ mit den Sommerfeldschen Integralformeln überein. Für $q=3$ und $n=0$ erhalten wir 


$$
\begin{aligned}
& H_{0}^{(1)}(3 ; r)=-i \frac{e^{i r}}{r}, \\
& H_{0}^{(2)}(3 ; r)=i \frac{e^{-i r}}{r} .
\end{aligned}
$$

Wir bilden daher

Definition 2. Die Funktionen

$$
\begin{aligned}
& H_{n}^{(1)}(q ; r)=-2 i^{-n} \frac{\omega_{q-1}}{\omega_{q}} \int_{1+0 i}^{1+\infty i} e^{i r t} P_{n}(q ; t)\left(1-t^{2}\right)^{\frac{q-3}{2}} d t, \\
& H_{n}^{(2)}(q ; r)=2 i^{-n} \frac{\omega_{q-1}}{\omega_{-1}} \int_{-1+\infty i}^{i+\infty} e^{i r t} P_{n}(q ; t)\left(1-t^{2}\right)^{\frac{q-3}{2}} d t
\end{aligned}
$$

heissen Hankelfunktionen erster und zweiter Art der Ordnung $n$ und Dimension $q$.

Im Vergleich mit Definition 1 folgt unmittelbar durch Zusammenlegen der Integrationswege

$$
H_{n}^{(1)}(q ; r)+H_{n}^{(2)}(q ; r)=2 J_{n}(q ; r) .
$$

Setzen wir noch

$$
H_{n}^{(1)}(q ; r)-H_{n}^{(2)}(q ; r)=2 i N_{n}(q ; r),
$$

so erhalten wir auch die Neumannschen Funktionen (die häufig mit $Y_{n}$ bezeichnet werden) der Ordnung $n$ und Dimension $q$. Die Normierungen sind dabei so gewählt, dass die Funktionen $J_{n}$ und $N_{n}$ für (reelle) $r$ reell sind. Die Besselfunktionen sind darüber hinaus ganze Funktionen der Veränderlichen $r$.

Die Funktion

$$
W_{n}(x)=\frac{i^{q-1}}{\omega_{q-1}} \int_{\Sigma_{q}\left(\xi^{\prime}\right)} e^{-x \cdot \eta} S_{n}(\eta) d \omega_{q}(\eta)
$$

existiert nach Satz 7 für $x \cdot \xi^{\prime}>0$. Bei festem $\xi^{\prime}$ können wir dann auch unter dem Integral differenzieren und erhalten wegen $\eta^{2}=1$

$$
\Delta W_{n}-W_{n}=0 .
$$

Andererseits ist nach Satz 7

$$
W_{n}(x)=K_{n}(q ; r) S_{n}(\xi)
$$

mit 


$$
K_{n}(q ; r)=\int_{1}^{\infty} e^{-r t} P_{n}(q ; t)\left(t^{2}-1\right)^{\frac{q-3}{2}} d t .
$$

Wir bilden daher

Definition 3. Die Funktionen

$$
K_{n}(q ; r)=\int_{1}^{\infty} e^{-r t} P_{n}(q ; t)\left(t^{2}-1\right)^{\frac{q-3}{2}} d t
$$

heissen Kelvinfunktionen der Ordnung $n$ und Dimension $q$.

Durch Vergleich der Integraldarstellungen aus Definition 2 und Definition 3 erhalten wir

$$
\begin{aligned}
H_{n}^{(1)}(q ; i r) & =i^{1-q-n} \frac{2 \omega_{q-1}}{\omega_{q}} K_{n}(q ; r), \\
H_{n}^{(2)}(q ;-i r) & =i^{n+q-1} \frac{2 \omega_{q-1}}{\omega_{q}} K_{n}(q ; r) .
\end{aligned}
$$

Bei diesen Beziehungen wird die Funktion $\left(1-t^{2}\right)^{(q-3) / 2}$ in der von -1 bis +1 aufgeschnittenen Ebene so definiert, dass an der Oberseite des Schnittes $\left(1-t^{2}\right)^{(q-3) / 2}$ positiv ist. Die Relationen (14) folgen dann durch Deformation des Integrationsweges.

Durch die Benutzung der verallgemeinerten Formel von Rodrigues aus der Theorie der Kugelfunktionen lassen sich die Hankel- und Kelvinfunktionen der Dimension $q$ durch elementare Umformungen auf die bekannten zweidimensionalen Funktionen zurückführen. Wir verzichten hier auf die Einzelheiten, da diese Rechnungen im Falle der Besselfunktionen (§3) bereits durchgeführt wurden (vgl. (3.19)), und beschränken uns darauf, die Vorteile des neuen Zugangs durch weitere Erläuterungen der zentralen Beziehungen dieses Abschnitts zu demonstrieren.

Zunächst bedeutet (1) für $\xi \cdot \xi^{\prime}>0 ; x=|x| \xi$

$$
(-1)^{j-1} \frac{2 i^{-n}}{\omega_{q}} \int_{\Omega_{q}^{(j)}\left(\xi^{\prime}\right)} e^{i x^{*} \cdot \eta} S_{n}(\eta) d \omega_{q}(\eta)=H_{n}^{(j)}(q ;|x|) S_{n}(\xi) .
$$

Setzen wir nun $\xi^{\prime}=\varepsilon_{q}$, und benutzen wir die Parameterdarstellung

$$
\eta=\sqrt{1-y^{2}} \varepsilon_{q}-y_{(q-1)} ; \quad y_{(q-1)} \in E_{q-1} ;
$$

für $\Omega_{q}^{(j)}\left(\varepsilon_{q}\right)$, so wird

$$
d \omega_{q}(\eta)=\frac{d \pi_{q-1}(y)}{\sqrt{1-y^{2}}}
$$


A. I. 415

Setzen wir weiterhin

$$
x=a \varepsilon_{q}+x_{(q-1)}, \quad x_{(q-1)}=x^{*},
$$

so wird aus (15) bis auf die Normierungsfaktoren

$$
\int_{E_{q-1}} e^{i a \sqrt{1-y^{2}}} e^{-i x^{*} \cdot y} S_{n}\left(\sqrt{1-y^{2}} \varepsilon_{q}-y\right) \frac{d \pi_{q-1}}{\sqrt{1-y^{2}}} .
$$

Spezialisieren wir weiter

$$
S_{n}(\eta)=P_{n}\left(q ; \varepsilon_{q} \cdot \eta\right)
$$

so ergibt sich für das Integral (19)

$$
\int_{E_{q-1}} e^{i a \sqrt{1-y^{2}}} e^{-i x^{*} \cdot y} P_{n}\left(q ; \sqrt{1-y^{2}}\right) \frac{d \pi_{q-1}}{\sqrt{1-y^{2}}} .
$$

Mit

$$
y=r \cdot \eta ; \quad 0 \leqq r<\infty ; \quad \eta \in \Omega_{q-1} ;
$$

wird aus (21) weiter

$$
\int_{0}^{\infty} e^{i a \sqrt{1-r^{2}}} P_{n}\left(q ; \sqrt{1-r^{2}}\right)\left(\int_{\Omega_{q-1}} e^{-i r x^{*} \cdot \eta} d \omega_{q-1}(\eta)\right) \frac{r^{q-2} d r}{\sqrt{1-r^{2}}} .
$$

Nun ist nach $\S 3$ mit $|x|=\sqrt{a^{2}+b^{2}}$ und $\left|x_{(q-1)}\right|=x^{*}=b$

$$
\frac{1}{\omega_{q-1}} \int_{\substack{\Omega_{q-1}\\}} e^{-i r x^{*} \cdot \eta} d \omega_{q-1}(\eta)=J_{0}(q-1 ; b r) .
$$

Somit folgt insgesamt

Lemma 8. Für $a>0, b>0$ ist mit $q \geqq 3$

$$
\begin{gathered}
(-1)^{j-1} \frac{2 i^{-n} \omega_{q-1}}{\omega_{q}} \int_{0}^{\infty} e^{i a v^{\prime} \overline{1-r^{2}}} J_{0}(q-1 ; r b) P_{n}\left(q ; \sqrt{1-r^{2}}\right) \frac{r^{q-2} d r}{\sqrt{1-r^{2}}} \\
=H_{n}^{(j)}\left(q ; \sqrt{a^{2}+b^{2}}\right) P_{n}\left(q ; \frac{a}{\sqrt{a^{2}+b^{2}}}\right) .
\end{gathered}
$$

wobei stets $\operatorname{Im}\left(\sqrt{1-r^{2}}\right) \geqq 0$ gilt, und für $j=1 \operatorname{Re}\left(\sqrt{1-r^{2}}\right) \geqq 0$ und für $j=2 \quad \operatorname{Re}\left(\sqrt{1-r^{2}}\right) \leqq 0 \quad z u$ setzen $i$ st.

Im Falle $q=3 ; n=0$ wird diese Beziehung als die Formel von Sommerfeld bezeichnet. 
Ausgangspunkt weiterer Integralbeziehungen ist die Formel (15) in der Form $\left(x_{q}>0\right)$

$$
\begin{gathered}
(-1)^{j-1} \frac{2 i^{-n}}{\omega_{q}} \int_{E_{q-1}} e^{i\left(x_{q}{\sqrt{1-y^{2}}}^{-x} \cdot y\right)} S_{n}\left(\sqrt{1-y^{2}} \varepsilon_{q}-y\right) \frac{d \pi_{q-1}(y)}{\sqrt{1-y^{2}}} \\
=H_{n}^{(j)}(q ;|x|) S_{n}\left(\frac{x}{|x|}\right)
\end{gathered}
$$

mit

$$
\begin{aligned}
& j=1 \quad \text { für } \quad 0 \leqq \arg \sqrt{1-y^{2}} \leqq \frac{\pi}{2}, \\
& j=2 \quad \text { für } \quad \frac{\pi}{2} \leqq \arg \sqrt{1-y^{2}} \leqq \pi .
\end{aligned}
$$

Eine analoge Beziehung erhalten wir aus (10) und (12) in der Form

$$
\begin{gathered}
\frac{1}{\omega_{q-1}} \int_{E_{q-1}} e^{-x_{q} \sqrt{1+y^{2}}-i x \cdot y} S_{n}\left(\sqrt{1+y^{2}} \varepsilon_{q}+i y\right) \frac{d \pi_{q-1}(y)}{\sqrt{1+y^{2}}} \\
=K_{n}(q ;|x|) S_{n}\left(\frac{x}{|x|}\right) .
\end{gathered}
$$

Durch Wahl verschiedener Kugelfunktionen und durch verschiedene Zusammenfassungen der mehrfachen Integrationen lassen sich aus den beiden Beziehungen viele Integralformeln ableiten. Wir betrachten als Beispiel einige Relationen, die zuerst von Sonine und Gegenbauer angegeben wurden. Zur Erläuterung dieser Gesetzmässigkeiten wollen wir uns auf den Fall $n=0$ beschränken.

Wir betrachten zunächst das Integral $\left(x_{q}>0\right)$

$$
\frac{1}{\omega_{q-1}} \int_{E_{q-1}} e^{-x_{q} \sqrt{1-b^{2}+y^{2}}-i x \cdot y} \frac{d \pi_{q-1}(y)}{\sqrt{1-b^{2}+y^{2}}}
$$

Für $1-b^{2}>0$ ergibt sich durch die Substitution $y=\sqrt{1-b^{2}} y^{\prime}$

$$
\begin{aligned}
& \left(1-b^{2}\right)^{\frac{q-2}{2}} \frac{1}{\omega_{q-1}} \int_{E_{q-1}} e^{-\sqrt{1-b^{2}}\left(x_{q} \sqrt{1+\left(y^{\prime}\right)^{2}}+i x \cdot y^{\prime}\right)} \frac{d \pi_{q-1}\left(y^{\prime}\right)}{\sqrt{1-\left(y^{\prime}\right)^{2}}} \\
& =\left(1-b^{2}\right)^{\frac{q-2}{2}} K_{0}\left(q ; \sqrt{1-b^{2}}|x|\right) \text {. }
\end{aligned}
$$

Für $1-b^{2}<0$ setzen wir $y=\sqrt{b^{2}-1} y^{\prime}$ und 


$$
\begin{gathered}
\sqrt{1-b^{2}+y^{2}}=-i \sqrt{b^{2}-1} \sqrt{1-\left(y^{\prime}\right)^{2}}, \\
0 \leqq \arg \sqrt{1-\left(y^{\prime}\right)^{2}} \leqq \frac{\pi}{2}
\end{gathered}
$$

Dann wird aus (27)

$$
\begin{gathered}
\left(b^{2}-1\right)^{\frac{q-2}{2}} \frac{2 i}{\omega_{q}} \int_{E_{q-1}} e^{i \sqrt{b^{2}-1}\left(\sqrt{1-\left(y^{\prime}\right)^{2}} x_{q}-x \cdot y^{\prime}\right)} \frac{d \pi_{q-1}\left(y^{\prime}\right)}{\sqrt{1-\left(y^{\prime}\right)^{2}}} \\
=\left(b^{2}-1\right)^{\frac{q-2}{2}} i H_{0}^{(1)}\left(q ; \sqrt{b^{2}-1}|x|\right) .
\end{gathered}
$$

Unter Beachtung der aus (14) folgenden Beziehung

$$
H_{0}^{(1)}(q ; i r)=\frac{2 \omega_{q-1}}{\omega_{q}} i^{1-q} K_{0}(q ; r)
$$

lassen sich die obigen Integrale für reelle $c$ und $0 \leqq \arg \sqrt{c-y^{2}} \leqq \tau / 2$ zusammenfassen zu

$$
\frac{2}{\omega_{q}} \int_{E_{q-1}} e^{i\left(\sqrt{c-y^{2}}-x \cdot y\right)} \frac{d \pi_{q-1}(y)}{\sqrt{c-y^{2}}}=c^{\frac{q-2}{2}} H_{0}^{(1)}(q ; \sqrt{c}|x|)
$$

mit $x_{q}>0$ und $0 \leqq \arg \sqrt{c} \leqq \pi / 2$. Beide Seiten dieser Gleichung sind als Funktionen von $c$ stetig für $q \geqq 3,|x|>0$, integrierbar für $q=2$, $|x|>0$, so dass die nun folgenden Vertauschungen der Reihenfolge der Integrationen gerechtfertigt sind.

Das Integral über $E_{q-1}$ können wir in mehrere Teile aufspalten, indem wir die Veränderlichen gruppenweise zusammenfassen.

Wir setzen dazu

$$
y_{(q-1)}=y_{(r-1)}+z_{(q-r)}
$$

mit $y_{(r-1)} \in E_{r-1}$ und $z_{(q-r)} \in E_{q-r}^{\perp}$. Dabei bezeichnen wir mit $E_{q-r}^{\perp}$ den von den Vektoren $\varepsilon_{r}, \varepsilon_{r+1}, \ldots, \varepsilon_{q-1}$ aufgespannten euklidischen Raum von $q-r$ Dimensionen.

Es wird dann

$$
\begin{aligned}
& \frac{2}{\omega_{q}} \int_{E_{q-1}} e^{i\left(\sqrt{1-y^{2}} x_{q}-x \cdot y\right)} \frac{d \pi_{q-1}(y)}{\sqrt{1-y^{2}}} \\
& =\frac{2}{\omega_{q}} \int_{E_{r-1}} \int_{E_{q-r}^{\perp}} e^{i\left(x_{q} \sqrt{1-y^{2}-z^{2}}-x \cdot y-x \cdot z\right)} \frac{d \pi_{r-1}(y) \wedge d \pi_{q-r}(z)}{\sqrt{1-y^{2}-z^{2}}} .
\end{aligned}
$$


Setzen wir nun noch

$$
x=x_{(r-1)}+x_{(q-r)}+x_{q} \varepsilon_{q} ; \quad x_{(r-1)} \in E_{q-1} ; \quad x_{(q-r)} \in E_{q-r}^{\perp},
$$

so wird unter Benutzung von (31) aus (34)

$$
\frac{\omega_{r}}{\omega_{q}} \int_{E_{q-r}^{\perp}} e^{-i x \cdot z}\left(1-z^{2}\right)^{\frac{r-2}{2}} H_{0}^{(1)}\left(r ; \sqrt{1-z^{2}}\right) \sqrt{x_{q}^{2}+x_{(r-1)}^{2}} d \pi_{q-r}(z) .
$$

Durch Einführung von Polarkoordinaten in $E_{q-r}^{\perp}$ ergibt sich dann mit

$$
\begin{aligned}
a^{2}=x_{q}^{2}+x_{(r-1)}^{2} ; & b^{2}=x_{(q-r)}^{2} ; \\
x^{2}=a^{2}+b^{2} ; & t^{2}=z^{2}
\end{aligned}
$$

$\operatorname{aus}(36)$

$$
\omega_{r} \omega_{q-r} \int_{0}^{\infty} J_{0}(q-r ; b t) H_{0}^{(1)}\left(r ; a \sqrt{1-t^{2}}\right) t^{q-r-1}\left(1-t^{2}\right)^{\frac{r-2}{2}} d t .
$$

Zusammenfassend folgt daher

Lemma 9. Es sei $a>0 ; b>0$. Dann ist für $r=1,2,3, \ldots, q-1$

$$
\begin{gathered}
\frac{\omega_{r} \omega_{q-r}}{\omega_{q}} \int_{0}^{\infty} J_{0}(q-r ; b t) H_{0}^{(1)}\left(r ; a \sqrt{1-t^{2}}\right) t^{q-r-1}\left(1-t^{2}\right)^{\frac{r-2}{2}} d t \\
=H_{0}^{(1)}\left(q ; \sqrt{a^{2}+b^{2}}\right) .
\end{gathered}
$$

Die in diesem Abschnitt dargelegten Integraldarstellungen sind somit eine Zusammenfassung vieler Beziehungen, die bisher isoliert betrachtet wurden, und die vielfach noch nicht formuliert sind.

Durch Benutzung der Theorie der mehrdimensionalen Fourier-Entwicklungen lassen sich weitere Relationen ableiten, die einer späteren Darstellung vorbehalten bleiben sollen.

\section{§ 7. Zusammenfassung}

Der hier vorgelegte Zugang zur Theorie der Besselfunktionen in beliebig vielen Dimensionen unterscheidet sich methodisch von dem klassischen Aufbau Watsons durch die konsequente Benutzung mehrdimensionaler Methoden sowohl im Bereich der reellen als auch der komplexen Analysis.

Die vorgeschlagenen Bezeichnungsweisen tragen dem Umstand Rechnung, dass sich viele Strukturen der Besselfunktionen mit Hilfe der Dimensionszahl so formulieren lassen, dass ihre Anwendung erleichtert wird. 
Die enge Verknüpfung der Theorie der Besselfunktionen mit den Kugelfunktionen erscheint vom Standpunkt der Theorie der partiellen Differentialgleichungen in beliebigen Dimensionen naturgemäss [3].

Die in höheren Dimensionen auftretenden, topologisch bedingten Singularitäten der Parametrisierungen der Sphären belasten explizite Darstellungen mit Hilfe von Polarkoordinaten durch Schwierigkeiten, die nicht durch das Problem bedingt sind. Eine Methode, die weitgehend ohne Festlegung der Parameter auskommt, muss daher wesentliche Vorteile der Formulierung und Beweistechnik bringen.

So wird die Übertragung der Sommerfeldschen Theorie auf mehr als zwei Dimensionen erst möglich, wenn man das Oberflächenelement der Einheitssphären als Differentialform der kartesischen Koordinaten auffasst. Damit wird auch der Übergang ins Komplexe leicht durchführbar.

Die als Beispiele angegebenen Integralbeziehungen und Reihenentwicklungen sind in anderer Formulierung bekannt. Hier liegt der entscheidende Vorteil in der Tatsache, dass sie aus einigen wenigen Formeln durch Spezialisierung oder Anwendung einfacher Grundstrukturen, wie der Vollständigkeit der Kugelfunktionen, folgen. Die sich aus den Gesetzmässigkeiten der mehrdimensionalen Fourier-Entwicklungen ergebenden Relationen wurden nicht explizit erwähnt.

Der klassische Aufbau benutzt die Methoden der eindimensionalen komplexen Analysis zur Darstellung einer Theorie der Besselfunktionen von beliebiger komplexer Ordnung und analysiert die sich daraus ergebenden mehrdeutigen Lösungen. Der hier vorgeschlagene Aufbau untersucht nur reguläre, eindeutige Lösungen ganzzahliger Ordnungen. Eine Untersuchung mehrdeutiger Lösungen steht noch aus.

Rhein.-Westf. Technische Hochschule

Aachen, Deutschland 


\section{Literatur}

[1] MÜLleR, CL.: Spherical harmonics. - Lecture Notes in Mathematics 17, SpringerVerlag, Berling / Heidelberg / New York, 1966.

[2] -"- Über die ganzen Lösungen der Wellengleichung. Nach einem Vortrag von G. Herglotz. - Math. Ann. 124, 1951/1952, S. 235-264.

[3] NiEmeyer, H.: Lokale und asymptotische Eigenschaften der Lösungen der Helmholtzschen Schwingungsgleichung. - J.-ber. Deutsch. Math.-Verein. 65,1962 , S. $1-44$.

[4] Sommer, F.: Über die Integralformeln in der Funktionentheorie mehrerer komplexer Veränderlichen. - Math. Ann. 125, 1952/1953, S. 172-182.

[5] Sommerfeld, A.: Mathematische Theorie der Diffraction. - Math. Ann. 47, 1896, S. $317-374$.

[6] - - Die Greensche Funktion der Schwingungsgleichung. - J.-ber. Deutsch. Math.-Verein. 21, 1912, S. 309-353.

[7] Watson, G. N.: A treatise on the theory of Bessel functions. - [Zweite Auflage.] The University Press, Cambridge, 1944. 